November 1992

\title{
New Perspectives on the BRST-algebraic Structure of String Theory
}

\author{
Bong H. Lian \\ University of Toronto \\ Department of Mathematics \\ Toronto, Ontario, Canada M5S $1 A 1$ \\ Gregg J. Zuckerman円 \\ Yale Univer sity \\ and Department of Mathematics \\ New Haven, CT 06520, USA
}

\begin{abstract}
Motivated by the descent equation in string theory, we give a new interpretation for the action of the symmetry charges on the BRST cohomology in terms of what we call the Gerstenhaber bracket. This bracket is compatible with the graded commutative product in cohomology, and hence gives rise to a new class of examples of what mathematicians call a Gerstenhaber algebra. The latter structure was first discussed in the context of Hochschild cohomology theory [11]. Off-shell in the (chiral) BRST complex, all the identities of a Gerstenhaber algebra hold up to homotopy.

Applying our theory to the $\mathrm{c}=1$ model, we give a precise conceptual description of the BRST-Gerstenhaber algebra of this model. We are led to a direct connection between the bracket structure here and the anti-bracket formalism in BV theory [29]. We then discuss the bracket in string backgrounds with both the left and the right movers. We suggest that the homotopy Lie algebra arising from our Gerstenhaber bracket is closely related to the HLA recently constructed by Witten-Zwiebach. Finally, we show that our constructions generalize to any topological conformal field theory.
\end{abstract}

\footnotetext{
${ }^{1}$ Supported by NSF Grant DMS-9008459 and DOE Grant DE-FG0292ER25121.
} 


\section{Introduction}

One of the many successes of string theory is to provide a testing ground for new ideas in physics as well as in mathematics. Often times, a success story begins with the study of a certain concrete model in string theory (or the cousins thereof). A proper understanding of a special case leads to generalizations that often go far beyond the original context. In this paper, we hope to illustrate yet another such episode of the evolution of string theory.

The enormous success of the matrix model may be credited for the recent revival of string theory. This second coming of string theory marks yet another exciting moment in math/physics. It is perhaps too soon to give a historical review of this development, for we are still in the midst of it. We will however focus on one particular aspect of string theory - the BRST structure.

Given a conformal field theory of central charge $c$, one may obtain a consistent string background by coupling the conformal theory to both the Liouville theory, with central charge $26-c$, and to the conformal ghost system, with central charge -26 . The background so obtained may then be studied using CFT techniques, barring some subtleties coming from the Liouville sector. The simplest of such backgrounds is the two dimensional string theory, a.k.a. the $\mathrm{c}=1$ model, in which a single free boson is coupled to the Liouville field and the ghosts.

It is useful to think of a string background from a slightly more abstract point of view. Namely, we can regard a string background as having the form

$$
C F T \otimes \text { ghosts }
$$

The CFT here is a conformal field theory with central charge 26 . In the case of the $\mathrm{c}=1$ model, the CFT may now be viewed as a two dimensional target spacetime for the string. The symmetry of the target can be exploited to study the background. For example, this symmetry alone plays a crucial role in our calculation of the BRST cohomology of the $\mathrm{c}=1$ model [20]. (Other methods have also been used to study this problem [1].) This indicates that some of the detailed structures of the individual constituents of the CFT in (1.1) may be spared when one is interested just in the BRST structures of the background.

The second advantage of considering (1.1) abstractly is that it is easier to pose the question: what are the generic structures of all string backgrounds? The abstract setting frees us from some of the special features and the subtleties of the conformal theory which one couples to the Liouville field and the ghosts. Having said that, we will, from now on consider the string 
backgrounds of the form (1.1). Later in the paper we will return to the $\mathrm{c}=1$ model.

The goal of this paper is to show that for every string background, the BRST cohomology has, intrinsically, the algebraic structure known as a Gerstenhaber algebra. In the case of the $\mathrm{c}=1$ model, an important realization of this algebra is implicit in the work of Witten and Zwiebach[31]. Recently Y. Wu and C. Zhu have reanalysed the same realization in detail[32].

In section 1.1, we discuss some basic definitions and results in the theory of super chiral algebras. In section 2, we present the general construction of the Gerstenhaber bracket on the BRST cohomology, and discuss some fundamental properties of the bracket.

Let the dot product, $u \cdot v$, be defined by eqn (2.11). Let the bracket product, $\{u, v\}$, be defined by eqn (2.19). Let $|u|$ be the ghost number of $u$. Let $b_{0}$ be the zero mode of the anti-ghost field.

Theorem 2.2: On the chiral cohomology $H^{*}$, we have

(a) $u \cdot v=(-1)^{|u||v|} v \cdot u$

(b) $(u \cdot v) \cdot t=u \cdot(v \cdot t)$

(c) $\{u, v\}=-(-1)^{(|u|-1)(|v|-1)}\{v, u\}$

(d) $(-1)^{(|u|-1)(|t|-1)}\{u,\{v, t\}\}+(-1)^{(|t|-1)(|v|-1)}\{t,\{u, v\}\}+(-1)^{(|v|-1)(|u|-1)}\{v,\{t, u\}\}=0$

(e) $\{u, v \cdot t\}=\{u, v\} \cdot t+(-1)^{(|u|-1)|v|} v \cdot\{u, t\}$

(f) $b_{0}\{u, v\}=\left\{b_{0} u, v\right\}+(-1)^{|u|-1}\left\{u, b_{0} v\right\}$

(g) $\{\}:, H^{p} \times H^{q} \longrightarrow H^{p+q-1}$.

Definition 1.1 An abstract Gerstenhaber algebra $G^{*}$ is a $\mathbf{Z}$-graded vector space equipped with two bilinear multiplication operations, denoted by $u \cdot v$ and $\{u, v\}$ respectively, and satisfying the following assumptions:

(i) If $u$ and $v$ are homogeneous elements of degree $|u|$ and $|v|$ respectively, then $u \cdot v$ is homogeneous of degree $|u|+|v|$ and $\{u, v\}$ is homogeneous of degree $|u|+|v|-1$.

(ii) Identities (a) through (e) from Theorem 2.9 above hold for any triple of homogeneous elements $u, v$ and $t$ in $G^{*}$.

In section 3, we apply our general theory to the $\mathrm{c}=1$ model. We give a characterization of the full chiral cohomology algebra.

Let $H^{*}$ be the chiral cohomology of the $\mathrm{c}=1$ model. Let $H^{*}( \pm)$ be subspaces of $H^{*}$ defined by eqn (3.16).

Theorem 3.2 Let $H^{*}$ be the chiral cohomology of the $c=1$ model. Let $\mathcal{A}^{*}$ be the Gerstenhaber 
algebra $\mathbf{C}\left[x, y, \partial_{x}, \partial_{y}\right]$ (see Appendix B). Then the following holds:

(a) There is an exact sequence of Gerstenhaber algebras

$$
0 \longrightarrow H(-) \longrightarrow H \longrightarrow \mathcal{A} \longrightarrow 0
$$

where $H(-)$ is an ideal in which both products are identically zero.

(b) $H^{*}(+)$ is closed under the dot product, and it is canonically isomorphic to $\mathcal{A}^{*}$, as an associative algebra.

(c) Let $H(+)^{\sigma}$ be the restricted dual of $H(+)$ defined by the $\bullet$-algebra anti-involution $\sigma$ (section 3.5). Then the ideal $H(-)$ is isomorphic, as module over $H(+)$, to $H(+)^{\sigma}$.

(d) $H^{*}(+)$ is not closed under the bracket product. The sequence in (a) does not split as an exact sequence of graded Lie algebras (For more details on the bracket, see section 3.9).

(e) The projection map $H \longrightarrow \mathcal{A}$ intertwines $-b_{0}$ on $H$ and the differential operator

$$
\Delta=\frac{\partial}{\partial x} \frac{\partial}{\partial x^{*}}+\frac{\partial}{\partial y} \frac{\partial}{\partial y^{*}}
$$

on $\mathcal{A}$ where $x^{*}=\partial_{x}, y^{*}=\partial_{y}$.

The main purpose of Appendix $\mathrm{A}$ is to show that $H^{*}$ is generated, as a Gerstenhaber algebra, by four generators $x, y, \partial_{x}, \partial_{y}$. Moreover, we describe a basis of $H(-)$, which is dual and complementary to the basis of $H(+)$ given in (3.26), and hence show that $H(-)$ is an ideal with one generator $\left\{\partial_{x}, \partial_{y}\right\}$. The pairing between the two bases is $s l_{2}$ invariant. We show, as a consequence, that $H(-)$ is a subalgebra of $H$ with the zero product. This last assertion can also be drawn from [32], where the product is explicitly computed. We also describe the action of $b_{0}, x, y, \partial_{x}, \partial_{y}$ on $H(-)$ in terms of the above dual basis.

In Appendix B, we briefly review the classical examples of Gerstenhaber brackets and algebras. We also attempt to clarify the relationship between Gerstenhaber brackets and BatalinVilkovisky anti-brackets.

Acknowledgement: During the course of this work, we have benefitted from many fruitful conversations with Louis Crane, Igor Frenkel, James Lepowsky, Bill Massey, Greg Moore, Ronen Plesser, Jim Stasheff, Ed Witten, Yongchang Zhu and Barton Zwiebach.

\subsection{Chiral algebras: review}


In this section, we discuss the definition and some basic properties of the super chiral algebra (a.k.a vertex operator algebra in the math literature), which are relevant to our later discussion. We refer the readers to the original papers for more extensive discussions on the subject. The following definition can be found in [9]. It is a refinement of the definition of [2] [3] [22]. In the context of closed string theory, one should think of a chiral algebra as a substructure of the full state space of a conformal string background. In most cases, the full state space is larger than the chiral algebra itself.

Definition 1.2 A super chiral algebra consists of the following data: a vector space $V$ graded by the conformal weight $\Delta$ and the fermion number $|\cdot|$, two distinguished elements $\mathbf{1}$ (the vacuum) and $L$ (the Virasoro element), and a one-to-one linear map

$$
\phi \mapsto \phi(z)=\sum \phi_{n} z^{-n-\Delta_{\phi}}
$$

where $\phi_{n}$ is a linear operator in $V$ of weight $-n$, such that the product, $\phi(z) \psi$, is a Laurent series with coefficients in $V$. The data satisfy the following further conditions:

(a) (Cauchy-Jacobi identity) Any two fields $\phi(z), \psi(z)$ satisfy

$$
\begin{aligned}
& \operatorname{Res}_{w} \operatorname{Res}_{z-w}(\phi(z-w) \psi)(w) f(z, w) \\
& =\operatorname{Res}_{z} \operatorname{Res}_{w} \phi(z) \psi(w) f(z, w)-(-1)^{|\phi||\psi|} \operatorname{Res}_{w} \operatorname{Res}_{z} \psi(w) \phi(z) f(z, w)
\end{aligned}
$$

where $f$ is any Laurent polynomial in $z, w, z-w$. Note that the three residues above are taken around the contours with $|w|>|z-w|,|z|>|w|$ and $|w|>|z|$ respectively.

(b) The vacuum corresponds to the identity operator;

(c) The field $L(z)$ has the OPE

$$
L(z) L(w) \sim \frac{c / 2}{(z-w)^{4}}+\frac{2 L(w)}{(z-w)^{2}}+\frac{\partial L(w)}{z-w}
$$

and $\left(L_{-1} \phi\right)(z)=\partial \phi(z)$. The scalar $c$ is called the central charge of the chiral algebra.

Note: Typically physicists denote the operation $\operatorname{Res}_{w}($.$) by \int_{C_{0}} d w($.$) , where C_{0}$ is a simple contour surrounding the point 0 .

There are many identities that follows from the above definition. Some may be found in [9] 10]. Others can be found in [33] [18]. Most of the results that we need here will be derived directly from the Cauchy-Jacobi identity. For example, if we let $f$ be an arbitrary Laurent polynomial of just $w$, and $\phi(z)$ be a current of weight 1, then the Cauchy-Jacobi identity immediately implies 
Lemma 1.3 The charge $\phi_{0}=\operatorname{Res}_{z} \phi(z)$ is a derivation of the product $\psi(w) \chi$, i.e.

$$
\phi_{0}(\psi(w) \chi)-(-1)^{|\phi||\psi|} \psi(w)\left(\phi_{0} \chi\right)=\left(\phi_{0} \psi\right)(w) \chi
$$

Another immediate consequence of the Cauchy-Jacobi identity is obtained if we set $\psi=\mathbf{1}$ and $f(z, w)=(z-w)^{-1} g(w)$ where $g(w)$ is an arbitrary Laurent polynomial. In this case, we get $\left(\phi_{-\Delta_{\phi}} \mathbf{1}\right)(w)-\phi(w)=0$. Since $\phi \mapsto \phi(w)$ is injective, it follows that

$$
\phi_{-\Delta_{\phi}} 1=\phi
$$

Similarly we have, for $n>-\Delta_{\phi}$,

$$
\phi_{n} 1=0
$$

Notations: We write $A$ for the state corresponding to the field $A(z)$, whatever the form

of $A(z)$. Thus $\partial \phi$ and $c e^{\sqrt{2} \phi}$ mean the states corresponding to the fields $\partial \phi(z)$ and $c(z) e^{\sqrt{2} \phi}(z)$ respectively.

\section{BRST cohomology and the Gerstenhaber Bracket}

In this section, we construct the Gerstenhaber bracket on the chiral BRST complex (1.1). We show that there is a canonical Lie algebra homomorphism from the space of old physical states to the ghost number one BRST cohomology.

\subsection{Setting}

Consider the chiral algebra of the BRST complex:

$$
C^{*}=V \otimes \Lambda^{*}
$$

where $V$ is the chiral algebra of a conformal field theory with central charge 26 , and $\Lambda^{*}$ denotes the chiral conformal ghost system. This space has two standard integral gradings given by the ghost number (fermion number) and the conformal weight. The subspace of elements with ghost 
number $n$ is denoted $C^{n}$. We write the ghost number of an element $a$ as $|a|$. Every element $a$ of $C^{*}$ with weight $\Delta$ corresponds uniquely to a field operator

$$
a \mapsto a(z)=\sum a_{n} z^{-n-\Delta}
$$

where $a_{n}$ is a linear operator on $C^{*}$ and $a_{n}$ lowers the weight by $n$.

The chiral algebra $\Lambda^{*}$ is generated by a pair of fields $(b(z), c(z))$ of weight $(2,-1)$ and with OPE

$$
b(z) c(w) \sim \frac{1}{z-w} .
$$

The stress-energy of this system is given by

$$
L^{\Lambda}(z)=2 \partial b(z) c(z)+b(z) \partial c(z)
$$

Here we follow the usual physics convention that whenever two fields with the same formal variable $z$ are multiplied, the product actually denotes the normal ordered product. Since $V$ itself is a chiral algebra, it also has a stress-energy field which we denote $L^{V}(z)$.

The (chiral) BRST current is a primary field of weight 1 given by

$$
J(z)=\left(L^{V}(z)+\frac{1}{2} L^{\Lambda}(z)\right) c(z)+\frac{3}{2} \partial^{2} c(z)
$$

We denote the BRST charge $J_{0}$ by $Q$. The (chiral) BRST cohomology is denoted $H^{*}$.

The integral gradings on $C^{*}$ are rather special. The ghost number is given by the eigenvalues of the charge $F_{0}$ of the ghost number current

$$
F(z)=c(z) b(z)
$$

The conformal weight is given by the eigenvalues of $L_{0}$, the zero mode of the total stress energy field

$$
L(z)=L^{V}(z)+L^{\Lambda}(z)
$$

This field is $Q$-exact because

$$
[Q, b(z)]=L(z)
$$

In particular, we have $\left[Q, b_{0}\right]=L_{0}$, which implies that a BRST invariant state is $Q$-exact unless it has weight zero.

Throughout this paper, [,] will always mean the graded commutator in some $\mathbf{Z}$ graded associative algebra $\mathcal{A}$. Thus if $u, v$ are homogeneous elements of $\mathcal{A}$, we have

$$
[u, v]=u v-(-1)^{|u||v|} v u
$$

The vacuum of a chiral algebra will be denoted 1 . 


\subsection{The multiplicative structure}

Since $Q$ is the charge of a current, it acts on the chiral algebra $C^{*}$ by derivation (Lemma 1.3), i.e. for any two homogeneous element $u$ and $v$ in $C^{*}$,

$$
Q(u(z) v)=(Q u)(z) v+(-1)^{|u|} u(z)(Q v)
$$

In particular, the bilinear operation which we call the dot (•) product:

$$
u \cdot v \stackrel{\text { def }}{=} \operatorname{Res}_{z} \frac{u(z) v}{z}=\operatorname{Res}_{z} \operatorname{Res}_{w} \frac{u(z) v(w) \mathbf{1}}{z w}
$$

satisfies

$$
Q(u \cdot v)=(Q u) \cdot v+(-1)^{|u|} u \cdot(Q v)
$$

Thus, the dot product induces a product on the BRST cohomology. By eqn (1.2), we have $u \cdot \mathbf{1}=u=\mathbf{1} \cdot u$. Thus $\mathbf{1}$ is the identity with respect to the dot product. We claim that eqn $(2.11)$ defines a homotopy graded commutative associative algebra off-shell, i.e. on the complex $C^{*}$. This implies in particular that the product induces a graded commutative associative product on-shell, i.e. on $H^{*}$. The notion of an algebraic identity holding only up to homotopy is discussed in some work of Stasheff [26] 27] (see also [16]).

By the Cauchy-Jacobi identity and eqn (2.11), we have

$$
\begin{aligned}
u \cdot v & -(-1)^{|u||v|} v \cdot u \\
& =\operatorname{Res}_{w} \operatorname{Res}_{z-w} \frac{(u(z-w) v)(w) \mathbf{1}}{(1+(z-w) / w) w^{2}} \\
& =\sum_{i \geq 0}(-1)^{i} \operatorname{Res}_{w} \operatorname{Res}_{z-w} \frac{(u(z-w) v)(w) \mathbf{1}}{(z-w)^{-i} w^{i+2}} \\
& =\sum_{i \geq 0} \frac{(-1)^{i}}{i+1} \operatorname{Res}_{w} \operatorname{Res}_{z-w} L_{-1} \frac{(u(z-w) v)(w) \mathbf{1}}{(z-w)^{-i} w^{i+1}} \text { (integration by parts) } \\
& =\sum_{i \geq 0} \frac{(-1)^{i}}{i+1} \operatorname{Res}_{w} \operatorname{Res}_{z-w} \frac{\left(Q b_{-1}+b_{-1} Q\right)(u(z-w) v)(w) \mathbf{1}}{(z-w)^{-i} w^{i+1}} \\
& =Q m(u, v)+m(Q u, v)+(-1)^{|u|} m(u, Q v)
\end{aligned}
$$

where $m$ is a bilinear operation defined by

$$
m(u, v)=\sum_{i \geq 0} \frac{(-1)^{i}}{i+1} \operatorname{Res}_{w} \operatorname{Res}_{z-w} \frac{b_{-1}(u(z-w) v)(w) \mathbf{1}}{(z-w)^{-i} w^{i+1}}
$$


Eqn (2.13) says precisely that the dot product is homotopy graded commutative.

Now consider

$$
\begin{aligned}
(u \cdot v) \cdot t= & u \cdot(v \cdot t) \\
= & \operatorname{Res}_{w} \operatorname{Res}_{z-w} \frac{(u(z-w) v)(w) t}{(z-w) w}-\operatorname{Res}_{z} \operatorname{Res}_{w} \frac{u(z) v(w) t}{z w} \\
= & \operatorname{Res}_{z} \operatorname{Res}_{w} \frac{u(z) v(w) t}{(1-w / z) z w}+(-1)^{|u||v|} \operatorname{Res}_{w} \operatorname{Res}_{z} \frac{v(w) u(z) t}{(1-z / w) w^{2}} \\
& -\operatorname{Res}_{z} \operatorname{Res}_{w} \frac{u(z) v(w) t}{z w} \\
= & \sum_{i>0} \operatorname{Res}_{z} \operatorname{Res}_{w} \frac{u(z) v(w) t}{z^{i+1} w^{-i+1}}+(-1)^{|u||v|} \sum_{i \geq 0} \operatorname{Res}_{w} \operatorname{Res}_{z} \frac{v(w) u(z) t}{z^{-i} w^{i+2}} \\
= & \sum_{i \geq 0} \frac{1}{i+1} \operatorname{Res}_{z} \operatorname{Res}_{w} \frac{\left(L_{-1} u\right)(z) v(w) t}{z^{i+1} w^{-i}} \\
& +(-1)^{|u||v|} \sum_{i \geq 0} \frac{1}{i+1} \operatorname{Res}_{w} \operatorname{Res}_{z} \frac{\left(L_{-1} v\right)(w) u(z) t}{z^{-i} w^{i+1}}(\text { integration by parts }) \\
= & \sum_{i \geq 0} \frac{1}{i+1} \operatorname{Res}_{z} \operatorname{Res}_{w} \frac{\left(\left(Q b_{-1}+b_{-1} Q\right) u\right)(z) v(w) t}{z^{i+1} w^{-i}} \\
& +(-1)^{|u||v|} \sum_{i \geq 0} \frac{1}{i+1} \operatorname{Res}_{w} \operatorname{Res}_{z} \frac{\left(\left(Q b_{-1}+b_{-1} Q\right) v\right)(w) u(z) t}{z^{-i} w^{i+1}} \\
= & Q n(u, v, t)+n(Q u, v, t)+(-1)^{|u|} n(u, Q v, t)+(-1)^{|u|+|v|} n(u, v, Q t)
\end{aligned}
$$

where $n$ is a trilinear operation defined by

$$
\begin{aligned}
n(u, v, t)= & \sum_{i \geq 0} \frac{1}{i+1} \operatorname{Res}_{z} \operatorname{Res}_{w} \frac{\left(b_{-1} u\right)(z) v(w) t}{z^{i+1} w^{-i}} \\
& +(-1)^{|u||v|} \sum_{i \geq 0} \frac{1}{i+1} \operatorname{Res}_{w} \operatorname{Res}_{z} \frac{\left(b_{-1} v\right)(w) u(z) t}{z^{-i} w^{i+1}}
\end{aligned}
$$

Eqn (2.15) says that the dot product is homotopy associative. This proves our claim.

\subsection{The bracket structure}

Let's motivate the following construction by something well-known - the descent equations. Let $u(z)$ be a BRST invariant field of weight 0 . Since $L_{-1}=\left[Q, b_{-1}\right]$, it follows that

$$
\partial u(z)=\left(L_{-1} u\right)(z)=\left(Q b_{-1} u\right)(z)=\left[Q,\left(b_{-1} u\right)(z)\right]
$$


This is an example of a descent equation. Since the left hand side is a derivative, the coefficient of $z^{-1}$ is zero on both sides. Thus we have a current $\left(b_{-1} u\right)(z)$ whose charge

$$
\left(b_{-1} u\right)_{0}=\operatorname{Res}_{z}\left(b_{-1} u\right)(z)
$$

is BRST invariant. So if $v$ is a BRST invariant state, then so is $\left(b_{-1} u\right)_{0} v$. In the case of the $\mathrm{c}=1$ model, the formulas for this operation of the BRST invariant charges on the BRST invariant states have been worked out in detail 31].

However, we would like to understand this operation at a more conceptual level. The operation $\left(b_{-1} u\right)_{0} v$ is clearly linear in both $u$ and $v$. What is this bilinear operation on the BRST invariant states? What does it tell us about the cohomology? What if we extend the operation off-shell? These are the motivating questions that lead us to study the bilinear operation. We will introduce the notation

$$
\{u, v\}=(-1)^{|u|} \operatorname{Res}_{z}\left(b_{-1} u\right)(z) v=(-1)^{|u|} \operatorname{Res}_{w} \operatorname{Res}_{z-w}(b(z-w) u)(w) v
$$

for all $u, v$ in $C^{*}$. Note that this operation decreases the net ghost number by one, i.e. $\{\}:, C^{p} \times C^{q} \longrightarrow C^{p+q-1}$. The sign $(-1)^{|u|}$ is to make the bracket conform to the convention in [4]. We claim that

(i) $Q$ acts by derivation on $\{$,$\} ;$

(ii) $\{$,$\} satisfies skew commutativity and the Jacobi identity, up to homotopy;$

(iii) this bracket is a biderivation on the dot product, up to homotopy. Thus the bracket, together with the dot product, defines a Gerstenhaber algebra structure on the BRST cohomology $H^{*}$;

(iv) $b_{0}$ acts by derivation on $\{$,$\} . Thus the relative BRST cohomology is closed under the Ger-$ stenhaber bracket.

Let's compute the action of $Q$ on the bracket:

$$
\begin{aligned}
Q\{u, v\}= & (-1)^{|u|} \operatorname{Res}_{z}\left[Q,\left(b_{-1} u\right)(z)\right] v+(-1)^{|u|}(-1)^{|u|-1} \operatorname{Res}_{z}\left(b_{-1} u\right)(z) Q v \\
= & (-1)^{|u|} \operatorname{Res}_{z}\left(Q b_{-1} u\right)(z) v-(-1)^{|u|}(-1)^{|u|} \operatorname{Res}_{z}\left(b_{-1} u\right)(z) Q v \\
= & (-1)^{|u|-1} \operatorname{Res}_{z}\left(b_{-1} Q u\right)(z) v+(-1)^{|u|} \operatorname{Res}_{z}\left(L_{-1} u\right)(z) v \\
& +(-1)^{|u|-1}(-1)^{|u|} \operatorname{Res}_{z}\left(b_{-1} u\right)(z) Q v \\
= & \{Q u, v\}+(-1)^{|u|-1}\{u, Q v\}
\end{aligned}
$$

Note that we have used the fact that $\left(L_{-1} u\right)(z)$ is a total derivative, and hence has zero residue. This proves (i). 
Now consider the (graded) skew commutativity property of $\{$,$\} . From eqn (2.19), it is$ unclear how this property holds. What we need is the following elementary but crucial result.

Lemma 2.1 The following identity holds

$$
(-1)^{|u|}\{u, v\}=b_{0}(u \cdot v)-\left(b_{0} u\right) \cdot v-(-1)^{|u|} u \cdot\left(b_{0} v\right)
$$

A remark about the identity: the right hand side clearly measures the failure of $b_{0}$ to be a derivation of the dot product. The same idea first appears in the "anti-bracket" formalism, but in a seemingly different context. In [29], Witten showed that the Batalin-Vilkovisky equation can be formulated using a certain fundamental differential operator $\Delta$ in field space, together with an anti-bracket which measures the failure of $\Delta$ to be a derivation of an operator product. The $b_{0}$ operator here plays the role of $\Delta$ ! We will, in Theorem 3.2, make a precise connection between these two operators in the context of the $\mathrm{c}=1$ model.

The identity above is proved by the following calculation:

$$
\begin{aligned}
& b_{0}(u \cdot v)-\left(b_{0} u\right) \cdot v-(-1)^{|u|} u \cdot\left(b_{0} v\right) \\
& =\operatorname{Res}_{z} \operatorname{Res}_{w} \frac{b(z) u(w) v}{z^{-1} w}-(-1)^{|u|} \operatorname{Res}_{w} \operatorname{Res}_{z} \frac{u(w) b(z) v}{z^{-1} w}-\operatorname{Res}_{w} \operatorname{Res}_{z-w} \frac{(b(z-w) u)(w) v}{(z-w)^{-1} w} \\
& =\operatorname{Res}_{w} \operatorname{Res}_{z-w} \frac{(b(z-w) u)(w) v}{z^{-1} w}-\operatorname{Res}_{w} \operatorname{Res}_{z-w} \frac{(b(z-w) u)(w) v}{(z-w)^{-1} w} \quad(\text { Cauchy }- \text { Jacobi }) \\
& =\operatorname{Res}_{w} \operatorname{Res}_{z-w}(b(z-w) u)(w) v \\
& =(-1)^{|u|}\{u, v\}
\end{aligned}
$$

The skew commutativity property of $\{$,$\} now becomes immediately obvious following Lemma$ 2.1, at least on-shell. Let's consider the bracket off-shell. A simple calculation gives us

$$
\begin{aligned}
\{u, v\} & +(-1)^{(|u|-1)(|v|-1)}\{v, u\} \\
& =(-1)^{|u|-1}\left(Q m^{\prime}(u, v)-m^{\prime}(Q u, v)-(-1)^{|u|} m^{\prime}(u, Q v)\right)
\end{aligned}
$$

where $m^{\prime}$ is yet another bilinear operation defined by

$$
m^{\prime}(u, v)=b_{0} m(u, v)+m\left(b_{0} u, v\right)+(-1)^{|u|} m\left(u, b_{0} v\right)
$$

Thus off-shell, the bracket $\{$,$\} satisfies graded skew commutativity up to homotopy.$

We now consider the graded Jacobi identity. Once again, it is an easy exercise to show that

$$
\begin{aligned}
& \{\{u, v\}, t\}-\{u,\{v, t\}\}+(-1)^{(|u|-1)(|v|-1)}\{v,\{u, t\}\} \\
& =(-1)^{(|v|-1)}\left(Q n^{\prime}(u, v, t)+n^{\prime}(Q u, v, t)+(-1)^{|u|} n^{\prime}(u, Q v, t)\right. \\
& \left.+(-1)^{|u|+|v|} n^{\prime}(u, v, Q t)\right)
\end{aligned}
$$


where $n^{\prime}$ is a trilinear operation defined by

$$
n^{\prime}(u, v, t)=\operatorname{Res}_{z} z\left(\operatorname{Res}_{w} b_{-1}\left(b_{-1}^{2} u\right)(w) v\right)(z) t
$$

Since $b_{-1}^{2}=0$, it follows that $n^{\prime}$ is identically zero, i.e. the graded Jacobi identity holds exactly. This completes the proof of (ii).

Consider now the derivation property of the bracket.

$$
\begin{aligned}
\{u, v \cdot t\}= & (-1)^{|u|} \operatorname{Res}_{z} \operatorname{Res}_{w} \frac{\left(b_{-1} u\right)(z) v(w) t}{w} \\
= & (-1)^{|u|} \operatorname{Res}_{w} \operatorname{Res}_{z-w} \frac{\left(\left(b_{-1} u\right)(z-w) v\right)(w) t}{w} \\
& +(-1)^{|u|}(-1)^{(|u|-1)|v|} \operatorname{Res}_{w} \operatorname{Res}_{z} \frac{v(w)\left(b_{-1} u\right)(z) t}{w} \\
= & \{u, v\} \cdot t+(-1)^{(|u|-1)|v|} v \cdot\{u, t\}
\end{aligned}
$$

This proves that each $\{u, *\}$ is a graded derivation of the dot product. A similar statement is true for $\{*, t\}$ but only up to homotopy. More precisely, we have

$$
\begin{aligned}
& \{u \cdot v, t\}-u \cdot\{v, t\}-(-1)^{(|t|-1)|v|}\{u, t\} \cdot v \\
& =(-1)^{|u|+|v|-1}\left(Q n^{\prime \prime}(u, v, t)-n^{\prime \prime}(Q u, v, t)\right. \\
& \left.-(-1)^{|u|} n^{\prime \prime}(u, Q v, t)-(-1)^{|u|+|v|} n^{\prime \prime}(u, v, Q t)\right)
\end{aligned}
$$

where $n^{\prime \prime}$ is a trilinear operation defined by

$$
n^{\prime \prime}(u, v, t)=u \cdot m^{\prime}(v, t)-m^{\prime}(u \cdot v, t)+(-1)^{|t||v|} m^{\prime}(u, t) \cdot v
$$

This proves (iii).

Finally, we consider the action of $b_{0}$ on the bracket. Using Lemma 2.1 and the fact that $b_{0}^{2}=0$, we have

$$
b_{0}\{u, v\}=\left\{b_{0} u, v\right\}+(-1)^{|u|-1}\left\{u, b_{0} v\right\}
$$

This proves (iv). Let's summarize all the on-shell algebraic identities we have derived.

Theorem 2.2 On the cohomology $H^{*}$, we have

(a) $u \cdot v=(-1)^{|u||v|} v \cdot u$

(b) $(u \cdot v) \cdot t=u \cdot(v \cdot t)$

(c) $\{u, v\}=-(-1)^{(|u|-1)(|v|-1)}\{v, u\}$

(d) $(-1)^{(|u|-1)(|t|-1)}\{u,\{v, t\}\}+(-1)^{(|t|-1)(|v|-1)}\{t,\{u, v\}\}+(-1)^{(|v|-1)(|u|-1)}\{v,\{t, u\}\}=0$

(e) $\{u, v \cdot t\}=\{u, v\} \cdot t+(-1)^{(|u|-1)|v|} v \cdot\{u, t\}$

(f) $b_{0}\{u, v\}=\left\{b_{0} u, v\right\}+(-1)^{|u|-1}\left\{u, b_{0} v\right\}$

(g) $\{\}:, H^{p} \times H^{q} \longrightarrow H^{p+q-1}$. 
Some remarks about these results. The space $H^{0}$ is a strict commutative algebra - a well-known fact. Note that $H^{1}$ is closed under the bracket and hence is an ordinary Lie algebra. Moreover, every $H^{q}$ is a module over $H^{1}$ via the map $(g)$ in the case $p=1$. If one further restricts to the case $q=0$, one sees that the Lie algebra $H^{1}$ acts, by derivations, on the commutative algebra $H^{0}$. This crucial fact has been implicitly used in an effective way to determine the structure of $H^{0}$ in the $\mathrm{c}=1$ model 28 .

Note also that $\{$,$\} may be viewed as a super Lie bracket because it is compatible with the$ grading "ghost number - 1". In particular, we have $\left\{H^{r+1}, H^{s+1}\right\} \subset H^{r+s+1}$. The map $u \mapsto$ $\{u, *\}$, which essentially assigns to each BRST invariant state of weight zero the corresponding charge $\operatorname{Res}_{z}\left(b_{-1} u\right)(z)$, realizes the adjoint representation of the above super Lie algebra. This realization is never faithful because $\{\mathbf{1}, *\}$ is identically zero.

\subsection{The old physical states}

We now relate the Lie algebra structure on the old physical states to that of $H^{1}$.

Once again let $V$ be a chiral algebra with central charge $c=26$. The space $P(V)$ of the old physical states is the subspace of Virasoro primary states of weight 1 , modulo the states of the form $L_{n} v, n<0$. More precisely,

$$
\begin{aligned}
& P(V)=V[1]^{V i r_{+}} / N(V) \\
& N(V)=\left(V i r_{-} V\right) \cap V[1]^{V i r_{+}}
\end{aligned}
$$

Let $\phi(z)$ be any primary field of weight 1 . This means, in particular, that the charge $\phi_{0}$ is Vir invariant:

$$
\left[L^{V}(z), \phi_{0}\right]=0
$$

It follows that one has a well-defined bilinear operation

$$
\{,\}: V[1]^{V i r_{+}} \times V[1]^{V i r_{+}} \longrightarrow V[1]^{V i r_{+}}, \quad(\phi, \psi) \mapsto-\phi_{0} \psi
$$

Let's consider the properties of this operation. Applying the Cauchy-Jacobi identity, we get

$$
\{\phi, \psi\}=-\operatorname{Res}_{w} \operatorname{Res}_{z} \frac{\phi(w) \psi(z) \mathbf{1}}{z}
$$




$$
\begin{aligned}
& =\operatorname{Res}_{w} \operatorname{Res}_{z-w} \frac{(\psi(z-w) \phi)(w) \mathbf{1}}{(1+(z-w) / w) w}-\operatorname{Res}_{z} \operatorname{Res}_{w} \frac{\psi(z) \phi(w) \mathbf{1}}{z} \\
& =\sum_{i \geq 0}(-1)^{i} \operatorname{Res}_{w} \frac{\left(\psi_{i} \phi\right)(w) \mathbf{1}}{w^{i+1}} \quad\left(\operatorname{Res}_{w} \phi(w) \mathbf{1}=0\right)
\end{aligned}
$$

Now note that the $i=0$ term in the last sum is equal to $-\{\psi, \phi\}$. Thus we have

$$
\begin{aligned}
& \{\phi, \psi\}+\{\psi, \phi\} \\
& =\sum_{i>0}(-1)^{i} \operatorname{Res}_{w} \frac{\left(\psi_{i} \phi\right)(w) \mathbf{1}}{w^{i+1}} \\
& =L_{-1} \sum_{i>0} \frac{(-1)^{i}}{i} \operatorname{Res}_{w} \frac{\left(\psi_{i} \phi\right)(w) \mathbf{1}}{w^{i}} \quad \text { (integration by parts) }
\end{aligned}
$$

This implies that $\{$,$\} is skew-symmetric modulo N(V)$. Similarly, it is also easy to check that the bilinear operation factors through $N(V)$, and that it satisfies the Lie algebra Jacobi identity modulo $N(V)$. Thus $\{$,$\} is a Lie bracket on the space P(V)$. This Lie algebra structure is already known: see [7] and references therein.

It is well-known that there are two natural maps

$$
\nu_{1}, \nu_{2}: V[1]^{V i r_{+}} \longrightarrow H^{1}, H^{2}
$$

which send a field $\phi(z)$ to $c(z) \phi(z)$ and $\partial c(z) c(z) \phi(z)$ respectively. Let's assume that $V$, as a Virasoro representation, has an invariant bilinear pairing such that the induced pairing on $H^{*}$ is non-degenerate. Then we have a pairing preserving map

$$
\nu: V[1]^{V i r_{+}} \longrightarrow H^{1} \oplus H^{2}, \quad \phi \mapsto \frac{1}{\sqrt{2}}\left(\nu_{1} \phi \oplus \nu_{2} \phi\right)
$$

Since $N(V)$ lies in the kernel of the pairing on $V$, under the map $\nu$, the space $N(V)$ must be sent to zero. Thus the map $\nu$ and hence $\nu_{1}$ factors through $N(V)$. So we have the map

$$
\nu_{1}: P(V) \longrightarrow H^{1}
$$

We claim that this is a Lie algebra homomorphism. By definition (2.19), we have

$$
\begin{aligned}
\left\{\nu_{1} \phi, \nu_{1} \psi\right\} & =-\operatorname{Res}_{z}\left(b_{-1} \nu_{1} \phi\right)(z) \nu_{1} \psi \\
& =-\operatorname{Res}_{z}\left(b_{-1} c_{1} \phi\right)(z) c_{1} \psi \\
& =-\operatorname{Res}_{z} c_{1} \phi(z) \psi \\
& =\nu_{1}\{\phi, \psi\}
\end{aligned}
$$


which proves the claim. In case the map $\nu_{1}$ is injective (which happens whenever $N(V)$ coincides with the kernel of the pairing on $V$ ), such as in the case of the 26 dimensional bosonic string theory, we have a concrete realization of the Lie subalgebra $P(V)$ of $H^{1}$.

\section{The $\mathrm{c}=1$ model}

In this section, we will apply the machinery of the last section to obtain a characterization of the cohomology algebra of the $\mathrm{c}=1$ model.

Let's first review the operator formalism of the $c=1$ model. The model may be described as a theory in which a single free boson $X$ is coupled to the Liouville field $\phi$ and the conformal ghosts $(b, c)$. As we did in [21], we consider the case in which the cosmological constant is zero. For now, we restrict ourselves to the holomorphic part of the theory.

\subsection{What's known}

The free boson sector can be simply described by the field $X(z)$ with the OPE

$$
X(z) X(w) \sim-\ln (z-w)
$$

The operators in this sector are linear combinations of

$$
P(X) e^{i p X}(z)
$$

where the $P(X)$ are polynomials in the derivatives of $X(z)$, and $p$ is the momentum of the operator (3.2). The corresponding state space is a direct sum of highest weight representations $F(p)$ of the Heisenberg algebra:

$$
\left[\alpha_{n}, \alpha_{m}\right]=n \delta_{n+m, 0}
$$

where $i \partial X(z)=\sum \alpha_{n} z^{-n-1}$. The stress-energy field is

$$
L^{X}(z)=-\frac{1}{2}(\partial X(z))^{2}
$$


The Liouville sector can be described in a similar way - with $X$ replaced by $\phi$ everywhere except that the stress-energy of this sector is given by

$$
L^{\phi}(z)=-\frac{1}{2}(\partial \phi(z))^{2}+\sqrt{2} \partial^{2} \phi(z)
$$

We write $i \partial \phi(z)=\sum j_{n} z^{-n-1}$, where the $j_{n}$ represent a Heisenberg algebra just like the $\alpha_{n}$ do. Anticipating that we eventually will deal only with pure imaginary Liouville momenta, we denote the Liouville state space by $F(-i \alpha)$. The $\alpha$ will be restricted to real later. But for now, $\alpha$ is arbitrary.

The ghost sector $\Lambda^{*}$ has already been described, so we won't repeat it here. Now the BRST operator $Q$ acts on the spaces

$$
\begin{aligned}
& C^{*}(p, \alpha)=F(p) \otimes F(-i \alpha) \otimes \Lambda^{*} \\
& Q: C^{n}(p, \alpha) \longrightarrow C^{n+1}(p, \alpha)
\end{aligned}
$$

Thus we have one cochain complex for every pair of momenta $p, \alpha$. Later we will restrict the momenta to lie in a certain two dimensional even lattice. This restriction will land us back in the framework we considered in section 2 .

The cohomology of the complexes $C^{*}(p, \alpha)$ was first studied by us, in connection with the $c<1$ models 19 17. This cohomology problem has a number of other interesting applications. In [20], we applied the results to the $\mathrm{c}=1$ model. (Subsequently, other methods were also used to analyze the cohomology of the $c \leq 1$ models [四] [23].) Our cohomology results have also led to some later work [8] which gains new insights into the structure theory of the Feigin-Fuchs representations of the Virasoro algebra.

Our results on the $\mathrm{c}=1$ model have recently been given an interesting physical interpretation 28] in connection with the matrix model. The object that plays a key role in this interpretation is what's called "the ground ring." This is the subalgebra $H^{0}$ of the associative algebra $H^{*}$. The main tool that was used to determine the structure of the ground ring was the action coming from the tachyon fields. (Actually, this is secretly a part of the action of $H^{1}$ on $H^{0}$ via the Gerstenhaber bracket, as we shall see.) The symmetry of the $c=1$ model has been better understood by means of the ground ring. More recently, the role of the symmetry has been further clarified in the context of closed string theory [31.

Let's now return to the cohomology problem (see [20] [1] and references therein).

Theorem 3.1 The cohomology $H^{*}(p, \alpha)$ of the complex $C^{*}(p, \alpha)$ is given as follows:

(a) $H^{*}(p, \alpha)$ is nontrivial iff either $(i)(p, \alpha)$ lies in one of the two complex lines defined by 
$(\alpha-\sqrt{2}+p)(\alpha-\sqrt{2}-p)=0$; or $($ ii $)(p, \alpha)$ lies in the intersection of the even lattice $\mathcal{L}$ and the past-future cone $\mathcal{C}$ in the real pa-plane. Here $\mathcal{L}$ and $\mathcal{C}$ are defined by (Figure 1):

$$
\begin{aligned}
\mathcal{L}= & \{(p, \alpha) \mid p-\alpha \in \sqrt{2} \mathbf{Z}, p, \alpha \in \mathbf{Z} / \sqrt{2}\} \\
\mathcal{C}= & \left\{(p, \alpha) \in \mathbf{R}^{2} \mid \text { either }(\alpha-\sqrt{2}+p) \text { and }(\alpha-\sqrt{2}-p)\right. \\
& \text { both greater than zero(future }), \text { or both less than zero(past) }\}
\end{aligned}
$$

(b) The dimensions of the $H^{*}(p, \alpha)$ in case (i) are

$$
\operatorname{dim} H^{n}(p, \alpha)= \begin{cases}1 & \text { if } n=1,2 \\ 0 & \text { otherwise }\end{cases}
$$

In case (ii) (hence $(p, \alpha)$ lies in $\mathcal{L})$, we have $H^{n}(p, \alpha)=0$ unless $0 \leq n \leq 3$, and

$$
\begin{aligned}
& \operatorname{dim} H^{0}(p, \alpha)= \begin{cases}1 & \text { if }(p, \alpha) \text { in the past cone } \\
0 & \text { if }(p, \alpha) \text { in the future cone }\end{cases} \\
& \operatorname{dim} H^{1}(p, \alpha)= \begin{cases}2 & \text { if }(p, \alpha) \text { in the past cone } \\
1 & \text { if }(p, \alpha) \text { in the future cone }\end{cases} \\
& \operatorname{dim} H^{2}(p, \alpha)= \begin{cases}1 & \text { if }(p, \alpha) \text { in the past cone } \\
2 & \text { if }(p, \alpha) \text { in the future cone }\end{cases} \\
& \operatorname{dim} H^{3}(p, \alpha)= \begin{cases}0 & \text { if }(p, \alpha) \text { in the past cone } \\
1 & \text { if }(p, \alpha) \text { in the future cone }\end{cases}
\end{aligned}
$$

The states in case (i) are basically the tachyon states (and their duals).

\subsection{Witten's ground ring}

To return to the framework of section 2, we will restrict the momentum values $(p, \alpha)$ to the lattice $\mathcal{L}$. It is known that the space

$$
V=\oplus_{(p, \alpha) \in \mathcal{L}} F(p) \otimes F(-i \alpha)
$$

together with the grading coming from the spectrum of $L_{0}=L_{0}^{X}+L_{0}^{\phi}$, forms a chiral algebra. Thus the super chiral algebra

$$
C^{*}=V \otimes \Lambda^{*}
$$


is example of the situation we considered in section 2. In particular, the BRST cohomology $H^{*}$ of the complex $C^{*}$ has all the structures stated in Theorem 2.2. In particular, $H^{0}$ is a commutative algebra.

Witten proves that $H^{0}$ is a polynomial algebra with two generators, which are represented by

$$
\begin{aligned}
\mathcal{O}_{1 / 2,1 / 2} & =\left(c b+\frac{i}{\sqrt{2}}(\partial X-i \partial \phi)\right) e^{(i X-\phi) / \sqrt{2}} \\
\mathcal{O}_{1 / 2,-1 / 2} & =\left(c b-\frac{i}{\sqrt{2}}(\partial X+i \partial \phi)\right) e^{(-i X-\phi) / \sqrt{2}}
\end{aligned}
$$

Let's briefly recap his argument. First it is shown that there are two special derivations, which we denote $\delta_{ \pm}$, acting on the ground ring. In particular, they act on $\mathcal{O}_{1 / 2, \pm 1 / 2}$ by

$$
\begin{aligned}
& \delta_{ \pm} \mathcal{O}_{1 / 2, \pm 1 / 2}=1 \\
& \delta_{ \pm} \mathcal{O}_{1 / 2, \mp 1 / 2}=0
\end{aligned}
$$

This immediately implies that all the monomials generated by $\mathcal{O}_{1 / 2, \pm 1 / 2}$ are necessarily nonzero. Moreover, it is easy to see that all such monomials have distinct momenta. Specifically, the monomials $\mathcal{O}_{1 / 2,1 / 2}^{n} \cdot \mathcal{O}_{1 / 2,-1 / 2}^{m}$ have momenta $(p, \alpha)=\left(\frac{n-m}{\sqrt{2}}, \frac{-n-m}{\sqrt{2}}\right)$. Thus they must be linearly independent. Now by Theorem 3.1 part (b), case (ii), we see that the momenta are multiplicity free in ghost number zero. This proves that the above monomials exhaust all of $H^{0}$. To summarize, we have an isomorphism of commutative algebras

$$
\psi: H^{0} \longrightarrow \mathbf{C}[x, y], \quad \mathcal{O}_{1 / 2,1 / 2}, \mathcal{O}_{1 / 2,-1 / 2} \mapsto x, y
$$

In a more recent paper of Witten and Zwiebach [31], the structure of the cohomology algebra $H^{*}$ has become better understood. For example, it has been indicated that $H^{*}$ contains a subalgebra which is isomorphic to the polynomial super algebra

$$
\mathcal{A}=\mathbf{C}\left[x, y, \partial_{x}, \partial_{y}\right]
$$

where $x, y$ are bosonic and $\partial_{x}, \partial_{y}$ are fermionic. What can we say about the dot and bracket products on the full cohomology space $H^{*}$ ? This is the subject of the next discussion. It is also one of the main applications of our theory in section 2 . 


\subsection{Extending the map $\psi$}

The polynomial super algebra $\mathcal{A}$ above may be thought of as a space of polyvector fields on the $x y$-plane. It was known to Schouten [24] that the space of polyvector fields admits a bracket operation $\{$,$\} which extends the Lie bracket on 1-vector fields, and extends the action of the$ 1 -vector fields on the algebra of functions (0-vector fields). In the case of the graded algebra $\mathcal{A}$ , this bracket is uniquely characterized by the identities (see also Appendix B):

(i) $|x|=|y|=0 ;\left|\partial_{x}\right|=\left|\partial_{y}\right|=1$

(ii) $\{x, y\}=\left\{\partial_{x}, y\right\}=\left\{\partial_{y}, x\right\}=0$

(iii) $\left\{\partial_{x}, x\right\}=\left\{\partial_{y}, y\right\}=1$

(iv) $\{u, v\}=-(-1)^{(|u|-1)(|v|-1)}\{v, u\}$

(v) $\{u, v \cdot t\}=\{u, v\} \cdot t+(-1)^{(|u|-1)|v|} v \cdot\{u, t\}$

where $u, v, t$ are any polyvector fields. Thus, $\mathcal{A}$ becomes a Gerstenhaber algebra. We denote by $\mathcal{A}^{p}$ the subspace of $p$-vector fields.

Note that every 1 -vector field is uniquely determined by a derivation on the algebra of 0 vector fields $\mathcal{A}^{0}$. Similarly, every 2 -vector field $f \cdot \partial_{x} \cdot \partial_{y}$ is determined by the operation

$$
\left\{f \cdot \partial_{x} \cdot \partial_{y}, *\right\}: \mathcal{A}^{0} \longrightarrow \mathcal{A}^{1}
$$

Recall the homomorphism $\psi$ (see (3.11)). The crucial things to notice are that $\psi$ is an isomorphism, and that both $H^{*}$ and $\mathcal{A}^{*}$ have a bracket structure. We can therefore extend $\psi$ in such a way that makes the two brackets compatible, as follows.

Given a ghost number 1 class $u$ in $H^{1}$, we let $\psi u$ be the 1 -vector field satisfying

$$
\{\psi u, f\}=\psi\left\{u, \psi^{-1} f\right\}, \quad f \text { is any polynomial function }
$$

Note that the bracket on the left hand side is for $\mathcal{A}^{*}$, while the one on the right is for $H^{*}$. Because $\left\{u, \psi^{-1} f\right\}$ is in $H^{0}$, the right hand side is well-defined. The map has now been extended to $\psi: H^{1} \longrightarrow \mathcal{A}^{1}$.

Similarly, given a ghost number 2 class $v$, we let $\psi v$ be the 2 -vector field satisfying

$$
\{\psi v, f\}=\psi\left\{v, \psi^{-1} f\right\}
$$

Since $\psi$ is well-defined on $H^{1}$, the right hand side of eqn (3.15) makes sense. Now since there is no $\mathcal{A}^{3}$, we set $\psi H^{3}=0$. Therefore, $\psi$ is now defined on all of $H^{*}$. Note that $\psi$ obviously preserves the ghost number. 
Since $\mathcal{A}^{0}$ is generated by $x, y$, the value of $\psi u$ is determined by the cases $f=x, y$ in eqn (3.14). The same is true for $\psi v$ in eqn (3.15). We now use this observation together with Theorem 3.1 to understand the kernel and the cokernel of $\psi$.

\subsection{The structure of $\psi$}

Let's first introduce some notations. For every real number $\alpha_{0}$, we denote by $H^{n}\left(\alpha \geq \alpha_{0}\right)$ the sum of all $H^{n}(p, \alpha)$ with $(p, \alpha)$ in the lattice $\mathcal{L}$ and $\alpha \geq \alpha_{0}$. Similarly for $H^{n}\left(\alpha<\alpha_{0}\right)$ etc. We also write

$$
\begin{aligned}
& H(+)=H^{0}(\alpha \leq 0) \oplus H^{1}(\alpha \leq \sqrt{2} / 2) \oplus H^{2}(\alpha \leq \sqrt{2}) \\
& H(-)=H^{3}(\alpha \geq 2 \sqrt{2}) \oplus H^{2}(\alpha \geq 3 \sqrt{2} / 2) \oplus H^{1}(\alpha \geq \sqrt{2})
\end{aligned}
$$

The space $H(+)(H(-))$ is what Witten-Zwiebach called the plus (minus) states 31. Note that by Theorem 3.1, (see also Figure 2) $H^{0}(\alpha>0)$ and $H^{3}(\alpha<2 \sqrt{2})$ are both zero. Thus $H( \pm$ ) are actually two complementary subspaces of $H$.

We claim that $\psi$ is a Gerstenhaber algebra homomorphism, and that

$$
\begin{aligned}
\operatorname{ker} \psi & =H(-) \\
\operatorname{im} \psi & =\mathcal{A} \cong H(+) \quad \text { as } \bullet-\text { algebras }
\end{aligned}
$$

We prove these results in stages:

(i) $\psi H^{1}(\alpha \geq \sqrt{2})=\psi H^{2}(\alpha \geq 3 \sqrt{2} / 2)=0$;

(ii) $\psi(u \cdot v)=\psi(u) \cdot \psi(v)$;

(iii) $\psi\{u, v\}=\{\psi u, \psi v\}$;

(iv) $\psi$ is onto, and when restricted to $H(+)$, is a $\bullet$-algebra isomorphism.

Because $\psi H^{3}=0$ by definition, part (i) proves $H(-) \subset$ ker $\psi$. Since $H(+)$ is a complementary subspace of $H(-)$ in $H$, (iv) proves eqns (3.17). Parts (ii) and (iii) prove that $\psi$ is a Gerstenhaber algebra homomorphism.

Recall that the states $\mathcal{O}_{1 / 2, \pm 1 / 2}$ have Liouville momenta $\alpha=-\sqrt{2} / 2$. By definition (3.14) and by momentum conservation, we have

$$
\left\{\psi H^{1}(\alpha \geq \sqrt{2}), x \text { or } y\right\}=\psi\left\{H^{1}(\alpha \geq \sqrt{2}), \mathcal{O}_{1 / 2, \pm 1 / 2}\right\} \subset \psi H^{0}(\alpha \geq \sqrt{2} / 2)
$$


The last space is zero as noted earlier. The condition (3.18) implies that $\psi H^{1}(\alpha \geq \sqrt{2})=0$. Similarly, we have

$$
\begin{aligned}
\left\{\psi H^{2}(\alpha \geq 3 \sqrt{2} / 2), x \text { or } y\right\} & =\psi\left\{H^{2}(\alpha \geq 3 \sqrt{2} / 2), \mathcal{O}_{1 / 2, \pm 1 / 2}\right\} \\
& \subset \psi H^{1}(\alpha \geq \sqrt{2}) \\
& =0
\end{aligned}
$$

This implies that $\psi H^{2}(\alpha \geq 3 \sqrt{2} / 2)=0$, and hence completes the proof of part (i).

To prove part (ii), we need to check that it holds for $u \in H^{r}, v \in H^{s}$ for any $0 \leq r, s \leq 3$. But note that (ii) trivially holds whenever $r+s \geq 3$, for then $\mathcal{A}^{r+s}=0$ and $\psi H^{r+s}=0$. It holds for $r=0, s=0$ because $\psi$ restricted to $H^{0}$ is a $\bullet$-algebra isomorphism. Let consider the first nontrivial case $u \in H^{0}, v \in H^{1}$.

$$
\begin{aligned}
\{\psi(u \cdot v), f\} & =\psi\left\{u \cdot v, \psi^{-1} f\right\} \\
& =\psi\left(u \cdot\left\{v, \psi^{-1} f\right\}\right) \quad\left(\left\{H^{0}, H^{0}\right\}=0\right) \\
& \left.=\psi(u) \cdot \psi\left\{v, \psi^{-1} f\right\} \quad \text { (by the case } r=0, s=0\right) \\
& =\psi(u) \cdot\{\psi(v), f\} \quad\left(\text { by definition of } \psi \text { on } H^{1}\right) \\
& =\{\psi(u) \cdot \psi(v), f\} \quad\left(\left\{\mathcal{A}^{0}, \mathcal{A}^{0}\right\}=0\right)
\end{aligned}
$$

This implies that $\psi(u \cdot v)=\psi(u) \cdot \psi(v)$. Now all the remaining cases can be handled the same way. This proves part (ii).

Since $\left\{H^{r}, H^{s}\right\} \subset H^{r+s-1}$, part (iii) holds trivially in the case $r+s \geq 4$ or the case $r+s \leq 0$. In the case $r=0, s=1$, part (iii) follows directly from the definition of $\psi$ in eqn (3.14). In the case $r=0, s=3$, it holds for the following reason. By momentum conservation, $\left\{H^{3}, \mathcal{O}_{1 / 2, \pm 1 / 2}\right\} \subset$ $H^{2}(\alpha \geq 3 \sqrt{2} / 2)$. But the right hand side gets sent to zero by part (i). This implies that $\psi\left\{H^{3}, \mathcal{O}_{1 / 2, \pm 1 / 2}\right\}=0$, hence $\psi\left\{H^{3}, H^{0}\right\}=0$. Since $\psi H^{3}=0$, it follows that both sides of part (iii) are zero.

There are three remaining nontrivial cases to check: $(r, s)=(1,1),(0,2),(1,2)$. We will do the first one, while the rest are similar. Let $u, v \in H^{1}$. Then $\{u, v\} \in H^{1}$. Thus we have

$$
\begin{aligned}
\{\psi\{u, v\}, f\} & =\psi\left\{\{u, v\}, \psi^{-1} f\right\} \\
& \left.=\psi\left\{u,\left\{v, \psi^{-1} f\right\}\right\}-\psi\left\{v,\left\{u, \psi^{-1} f\right\}\right\} \quad \text { (by Jacobi id for } H^{*}\right) \\
& =\left\{\psi u, \psi\left\{v, \psi^{-1} f\right\}-\left\{\psi v, \psi\left\{u, \psi^{-1} f\right\} \quad \text { (by the case } r=0, s=1\right)\right. \\
& =\left\{\psi u,\{\psi v, f\}-\left\{\psi v,\{\psi u, f\} \quad \text { (by definition of } \psi \text { on } H^{1}\right)\right. \\
& \left.=\{\{\psi u, \psi v\}, f\} \quad \text { (by Jocobi id for } \mathcal{A}^{*}\right)
\end{aligned}
$$


This completes the proof of part (iii).

To prove the first half of part (iv), it is enough to show that the fermionic generators $\partial_{x}, \partial_{y}$ of $\mathcal{A}$ are in the image of $\psi$. This has basically been done already in 28] 31]. Consider the following BRST invariant states:

$$
Y_{1 / 2, \pm 1 / 2}^{+}=-c e^{( \pm i X+\phi) / \sqrt{2}}
$$

Let's compute $\psi Y_{1 / 2,1 / 2}^{+}$.

$$
\begin{aligned}
\left\{\psi Y_{1 / 2,1 / 2}^{+}, y\right\} & =\psi \operatorname{Res}_{z}\left(b_{-1} c e^{(i X+\phi) / \sqrt{2}}\right)(z) \mathcal{O}_{1 / 2,-1 / 2} \\
& =\psi \operatorname{Res}_{z} e^{(i X+\phi) / \sqrt{2}}(z) \mathcal{O}_{1 / 2,-1 / 2} \\
& =1 \\
\left\{\psi Y_{1 / 2,1 / 2}^{+}, x\right\} & =\psi \operatorname{Res}_{z} e^{(i X+\phi) / \sqrt{2}}(z) \mathcal{O}_{1 / 2,1 / 2} \\
& =0
\end{aligned}
$$

Therefore, we have

$$
\psi Y_{1 / 2,1 / 2}^{+}=\partial_{y}
$$

Similarly,

$$
\psi Y_{1 / 2,-1 / 2}^{+}=\partial_{x}
$$

This proves that $\psi$ maps onto $\mathcal{A}$.

It is now clear that under $\psi$, the monomials

$$
\left(\mathcal{O}_{1 / 2,1 / 2}\right)^{n} \cdot\left(\mathcal{O}_{1 / 2,-1 / 2}\right)^{m} \cdot\left(Y_{1 / 2,-1 / 2}^{+}\right)^{\nu} \cdot\left(Y_{1 / 2,1 / 2}^{+}\right)^{\mu}
$$

are sent to

$$
x^{n} \cdot y^{m} \cdot \partial_{x}{ }^{\nu} \cdot \partial_{y}{ }^{\mu}
$$

in $\mathcal{A}$, where $n, m$ are nonnegative integers and $\nu, \mu$ are 0 or 1 . Now by momentum counting and the multiplicity results in Theorem 3.1, we see that the monomials (3.26) form a basis of $H(+)$. In particular, as a graded $\bullet$-algebra, $H(+)$ is isomorphic to $\mathcal{A}$.

This completes the proof of all of our claims. To summarize, we have an exact sequence of Gerstenhaber algebras:

$$
0 \longrightarrow H^{*}(-) \hookrightarrow H^{*} \stackrel{\psi}{\longrightarrow} \mathcal{A}^{*} \longrightarrow 0
$$

Moreover, there is a splitting isomorphism $\mathcal{A}^{*} \stackrel{\sim}{\longrightarrow} H^{*}(+)$, as associative algebras. To simplify notations, we denote

$$
x=\mathcal{O}_{1 / 2,1 / 2}
$$




$$
\begin{aligned}
y & =\mathcal{O}_{1 / 2,-1 / 2} \\
\partial_{x} & =Y_{1 / 2,-1 / 2}^{+} \\
\partial_{y} & =Y_{1 / 2,1 / 2}^{+}
\end{aligned}
$$

It should become clear from the context when we write $x, y, \partial_{x}, \partial_{y}$, whether they live in $H(+)$ or in $\mathcal{A}$.

\subsection{The structure of the dot product}

The original goal of this chapter was to apply section 2 to understand the structure of the Gerstenhaber algebra $H^{*}$. This means that we must at least know how to describe the operations •, $\{\}:, H \times H \longrightarrow H$ in simple terms. We will first focus on the dot product.

We have already fully understood

$$
\text { • : } H(+) \times H(+) \longrightarrow H(+)
$$

Since we have established that $H(-)$ is an ideal in $H$, the problem is further reduced to studying

$$
\text { • : } H \times H(-) \longrightarrow H(-)
$$

i.e. studying $H(-)$ as a module over the algebra $H$. In the appendix, we show that

$$
H(-) \cdot H(-)=0
$$

To understand the dot product, it remains to study $H(-)$ as a module over the $\bullet$-algebra $H(+)$.

Since $H(+)$ is a polynomial algebra, it is graded by the degree. The subspace $H(+)[n]$ of polynomials of a fixed degree $n$ is of course finite dimensional. Let

$$
H(+)^{\prime}=\oplus H(+)[n]^{\prime}
$$

be the restricted dual of $H(+)$. Let $\sigma$ be the linear anti-involution of $H(+)$ defined by

$$
\begin{aligned}
\sigma(x) & =-x \\
\sigma(y) & =-y \\
\sigma\left(\partial_{x}\right) & =\partial_{x} \\
\sigma\left(\partial_{y}\right) & =\partial_{y} \\
\sigma(u \cdot v) & =\sigma(v) \cdot \sigma(u), \text { for all } u, v
\end{aligned}
$$


Then we can define an $H(+)$-module structure on $H(+)^{\prime}$ as follows: for any linear functional $\lambda \in H(+)^{\prime}$, and any $u, v \in H(+)$, we let

$$
(u \cdot \lambda)(v)=\lambda(\sigma(u) \cdot v)
$$

We denote this dual module by $H(+)^{\sigma}$. We claim that $H(-)$ is isomorphic to $H(+)^{\sigma}$ as $H(+)-$ modules.

First recall that on the the BRST complex, there is a non-degenerate bilinear pairing

$$
\langle,\rangle: C^{r}(p, \alpha) \times C^{3-r}(-p, 2 \sqrt{2}-\alpha) \longrightarrow \mathbf{C}
$$

which satisfies

$$
\left\langle\mathbf{1}, \partial^{2} c \partial c c e^{2 \sqrt{2} \phi}\right\rangle=2
$$

In terms of Figure 1, each point $(p, \alpha)$ of the lattice $\mathcal{L}$ is paired with its image $(-p, 2 \sqrt{2}-\alpha)$ under the reflection through the point $(0, \sqrt{2})$. With respect to this pairing, we have

$$
\begin{aligned}
\alpha_{n}^{\dagger} & =-\alpha_{-n} \\
j_{n}^{\dagger} & =-j_{-n}-2 i \sqrt{2} \delta_{n, 0} \\
c_{n}^{\dagger} & =c_{-n} \\
b_{n}^{\dagger} & =b_{-n}
\end{aligned}
$$

This implies that the BRST operator $Q$ is self-adjoint, and hence there is an induced nondegenerate pairing on the cohomology:

$$
\langle,\rangle: H^{r}(p, \alpha) \times H^{3-r}(-p, 2 \sqrt{2}-\alpha) \longrightarrow \mathbf{C}
$$

Notice that by definition (3.16), $H(+)$ pairs with $H(-)$ in a natural way. Thus we simply define the isomorphism

$$
H(-) \longrightarrow H(+)^{\prime}, \quad \lambda \mapsto\langle\lambda,\rangle
$$

The question is: does this map respect the action of $H(+)$ ? i.e. do we get, for any $u, v$ in $H(+)$,

$$
\langle u \cdot \lambda, v\rangle=\langle\lambda, \sigma(u) \cdot v\rangle ?
$$

To answer, it is enough to check this for the generators of $H(+): u=x, y, \partial_{x}, \partial_{y}$. We illustrate this in the simplest case $u=\partial_{y}$, the rest being similar but more tedious. 
By definition of the dot product (2.11), we have

$$
\begin{aligned}
\left\langle\partial_{y} \cdot \lambda, v\right\rangle & =\operatorname{Res}_{z}\left\langle-c e^{(i X+\phi) / \sqrt{2}}(z) \lambda, v\right\rangle \frac{1}{z} \\
& =-\sum_{n}\left\langle c_{-n}\left(e^{(i X+\phi) / \sqrt{2}}\right)_{n} \lambda, v\right\rangle \\
& =-\sum_{n}\left\langle\lambda,\left(e^{(i X+\phi) / \sqrt{2}}\right)_{-n} c_{n} v\right\rangle \\
& =\left\langle\lambda, \partial_{y} \cdot v\right\rangle
\end{aligned}
$$

But because $\sigma\left(\partial_{y}\right)=\partial_{y}$, we see that eqn (3.41) indeed checks out for $u=\partial_{y}$. The other cases are done in a similar way. Thus we have

$$
H(-) \cong H(+)^{\sigma}
$$

\subsection{The bracket structure}

To understand the bracket structure, let's consider the exact sequence of graded Lie algebras:

$$
0 \longrightarrow H(-) \longrightarrow H \stackrel{\psi}{\longrightarrow} \mathcal{A} \longrightarrow 0
$$

We would like to describe $H$ as an extension of the (graded) Lie algebra $\mathcal{A}$, by a (graded) module $H(-)$. (The bracket on $H(-)$ is zero by Appendix A.)

The first ingredient for describing the extension is the two-cocycle $\gamma$ corresponding to the sequence (3.44). This is a bilinear map $\gamma: \mathcal{A} \times \mathcal{A} \longrightarrow H(-)$ which can be computed quite easily. We will give the formula without going into the details. In terms of the canonical basis $x^{n} \cdot y^{m} \cdot \partial_{x}{ }^{\nu} \cdot \partial_{y}{ }^{\mu}$ of $\mathcal{A}$, we have

$$
\gamma\left(x^{n} \cdot y^{m} \cdot \partial_{x}{ }^{\nu} \cdot \partial_{y}{ }^{\mu}, x^{n^{\prime}} \cdot y^{m^{\prime}} \cdot \partial_{x}{ }^{\nu^{\prime}} \cdot \partial_{y}{ }^{\mu^{\prime}}\right)=\delta_{n, 0} \delta_{m, 0} \delta_{m^{\prime}, 0} \delta_{n^{\prime}, 0}\left\{\partial_{x}{ }^{\nu} \cdot \partial_{y}{ }^{\mu}, \partial_{x}{ }^{\nu^{\prime}} \cdot \partial_{y}{ }^{\mu^{\prime}}\right\}
$$

Note that the bracket on the right hand side is defined on $H$. The right hand side is not identically zero because $\left\{\partial_{x}, \partial_{y}\right\}$ is a non-zero element of $H(-)$ (see Appendix A).

We now describe the module $H(-)$. Since $\psi: H \longrightarrow \mathcal{A}$ is a projection map, every element in $\mathcal{A}$ has the form $\psi u$, for some cohomology class $u$ in $H(+)$. The action of $\mathcal{A}$ on the module $H(-)$ is then defined by

$$
(\psi u) \lambda=\{u, \lambda\}
$$


where $\lambda$ is in $H(-)$. We claim that $H(-)$ is isomorphic to the restricted dual module $\mathcal{A}^{\pi \circ \sigma}$ defined as follows. Let $\mathcal{A}^{\pi}$ be the twisted adjoint representation given by

$$
\pi(a) b=(-1)^{|a|-1}(\{a, b\}-2(\Delta a) \cdot b)
$$

for $a, b$ in $\mathcal{A}$. Here $\Delta$ is defined by eqn (3.59). Then $\mathcal{A}^{\pi \circ \sigma}$ is defined to be the $\sigma$-dual of $\mathcal{A}^{\pi}$. That is, $\mathcal{A}^{\prime}$ is the underlying space of $\mathcal{A}^{\pi \circ \sigma}$; and an element $a$ of $\mathcal{A}$ acts on an element $\chi$ of $\mathcal{A}^{\prime}$ by

$$
\langle a \chi, b\rangle=\langle\chi, \pi \circ \sigma(a) b\rangle
$$

To prove the claim, define the linear isomorphism

$$
\phi: H(-) \longrightarrow \mathcal{A}^{\pi \circ \sigma}=\mathcal{A}^{\prime} \quad \lambda \mapsto\left\langle\lambda, \psi^{-1}(\cdot)\right\rangle
$$

Note that $\phi$ is independent of the choice of the "inverse". We need to check that $\phi a \lambda=a \phi \lambda$, for all $a$ in $\mathcal{A}$ and $\lambda$ in $H(-)$. Equivalently, we can check that for all $u, v$ in $H(+)$,

$$
\langle\phi(\psi u) \lambda, \psi v\rangle=\langle\phi \lambda,(\pi \circ \sigma(\psi u)) \psi v\rangle
$$

It turns out that all we need to use is Lemma 2.1, the fact that under the pairing on $H, b_{0}^{\dagger}=b_{0}$, and that the map $\psi$ intertwines $b_{0}$ with $-\Delta$ (see section 3.8):

$$
\begin{aligned}
\langle\phi(\psi u) \lambda, \psi v\rangle & =\langle\{u, \lambda\}, v\rangle \\
& =\left\langle\lambda,(-1)^{|u|-1}\left(\left\{u^{\dagger}, v\right\}+2\left(b_{0} u^{\dagger}\right) \cdot v\right)\right\rangle \\
& =\left\langle\lambda,(-1)^{|\psi u|-1} \psi^{-1}(\{\sigma(\psi u), \psi v\}-2(\Delta \sigma(\psi u)) \cdot \psi v)\right\rangle \\
& =\left\langle\lambda, \psi^{-1}(\pi \circ \sigma(\psi u))(\psi v)\right\rangle \\
& =\langle\phi \lambda,(\pi \circ \sigma(\psi u))(\psi v)\rangle
\end{aligned}
$$

This proves our claim.

Thus we have shown that $H$, as a graded Lie algebra, is an extension of the Lie algebra of polyvector fields $\mathcal{A}$ by the dual module $\mathcal{A}^{\pi \circ \sigma}$. Moreover, this extension is characterized by the two-cocycle $\gamma$ above.

\subsection{The $b_{0}$ operator}


By virtue of Lemma 2.1, studying the operator

$$
b_{0}: H^{n} \longrightarrow H^{n-1}
$$

should allow us to better understand the bracket product. Let's first focus on $H(+)$.

Since there are no states of ghost number -1 , any polynomial $f$ in $H^{0}$ satisfies

$$
b_{0} f=0
$$

Similarly, we have for any two polynomials $f, g$,

$$
\{f, g\}=0
$$

By direct calculation using eqns (3.29), we also have

$$
\begin{aligned}
\left\{\partial_{x}, x\right\} & =\left\{\partial_{y}, y\right\}=1 \\
\left\{\partial_{x}, y\right\} & =\left\{\partial_{y}, x\right\}=0 \\
b_{0} \partial_{x} & =b_{0} \partial_{y}=0
\end{aligned}
$$

Thus by Lemma 2.1, we have

$$
\begin{aligned}
& \partial_{x} f=\left\{\partial_{x}, f\right\}=-b_{0}\left(f \cdot \partial_{x}\right) \\
& \partial_{y} f=\left\{\partial_{y}, f\right\}=-b_{0}\left(f \cdot \partial_{y}\right)
\end{aligned}
$$

That is, $b_{0}$ acts on the 1 -vector fields $H^{1}(+)$ by $-d i v$.

Finally, since $b_{0}$ kills both $\partial_{x}, \partial_{y}$, we have (Lemma 2.1)

$$
\begin{aligned}
\left\{\partial_{x}, \partial_{y}\right\} & =-b_{0}\left(\partial_{x} \cdot \partial_{y}\right) \\
& =-b_{0}\left(-\partial c c e^{\sqrt{2} \phi}\right) \\
& =c e^{\sqrt{2} \phi}
\end{aligned}
$$

On the 2-vector fields $H^{2}(+)$, we have

$$
\begin{aligned}
b_{0}\left(f \cdot \partial_{x} \cdot \partial_{y}\right) & =\left\{f, \partial_{x} \cdot \partial_{y}\right\}+f \cdot b_{0}\left(\partial_{x} \cdot \partial_{y}\right) \\
& =\partial_{y} f \cdot \partial_{x}-\partial_{x} f \cdot \partial_{y}-f \cdot\left\{\partial_{x}, \partial_{y}\right\}
\end{aligned}
$$

This completes the description of the $b_{0}$ operator on $H(+)$.

Note that because $\left\{\partial_{x}, \partial_{y}\right\} \in H(-)$, neither the $b_{0}$ operator nor the bracket stabilizes $H(+)$. However, since $b_{0}$ carries zero momenta, the definition (3.16) of $H(-)$ shows that $b_{0}$ preserves 
$H(-)$. Now because the dot product restricted to $H(-)$ is zero (see Appendix A), it follows that the bracket restricted to $H(-)$ is also zero.

Since $b_{0}^{\dagger}=b_{0}$ under the pairing between $H(+)$ and $H(-)$, we have $\left\langle b_{0} \lambda, v\right\rangle=\left\langle\lambda, b_{0} v\right\rangle$ for all $\lambda$ in $H(-)$ and $v$ in $H(+)$.

\subsection{Relation between $b_{0}$ and $\Delta$}

As remarked in section 2, $b_{0}$ plays a role analogous to that of certain differential operator $\Delta$, in the anti-bracket formalism (see [29]). If we now consider the latter in the case where the field space is the xy-plane, then $\Delta$ is acting in a certain algebra of polyvector fields on the plane. Here we take the algebra to be $\mathcal{A}=\mathbf{C}\left[x, y, \partial_{x}, \partial_{y}\right]$. Then we have

$$
\Delta=\frac{\partial}{\partial x} \frac{\partial}{\partial x^{*}}+\frac{\partial}{\partial y} \frac{\partial}{\partial y^{*}}
$$

where $x^{*}=\partial_{x}, y^{*}=\partial_{y}$. We claim that the homomorphism $\psi$ of Gerstenhaber algebras intertwines between $b_{0}$ and $-\Delta$, i.e. for every $\operatorname{BRST}$ class $u$, we have

$$
\psi\left(b_{0} u\right)=-\Delta(\psi u)
$$

Since $b_{0}$ stabilizes $H(-)$ which is the kernel of $\psi$, eqn (3.60) holds trivially when $u$ is in $H(-)$. So let's focus on $H(+)$. As before, $b_{0}$ kills $H^{0}$. On the other hand, $\Delta$ kills $\mathcal{A}^{0}$. On $H^{1}(+),-b_{0}$ acts as the divergence operator (3.56). But so does $\Delta$ on $\mathcal{A}^{1}$. Finally, $b_{0}$ acts on $H^{2}(+)$ by eqn (3.58). Since $\left\{\partial_{x}, \partial_{y}\right\}$ of $H$ gets sent to zero by $\psi$, we have

$$
\psi b_{0}\left(f \cdot \partial_{x} \cdot \partial_{y}\right)=\partial_{y} f \cdot x^{*}-\partial_{x} f \cdot y^{*}
$$

But the right hand side coincides with $-\Delta\left(f \cdot x^{*} \cdot y^{*}\right)$. This completes the proof of eqn (3.60).

To summarize our application of section 2 to the $c=1$ model, we have

Theorem 3.2 Let $H^{*}$ be the chiral cohomology of the $c=1$ model. Let $\mathcal{A}^{*}$ be the Gerstenhaber algebra $\mathbf{C}\left[x, y, \partial_{x}, \partial_{y}\right]$ (see Appendix B). Then the following holds:

(a) There is an exact sequence of Gerstenhaber algebras

$$
0 \longrightarrow H(-) \longrightarrow H \longrightarrow \mathcal{A} \longrightarrow 0
$$


where $H(-)$ is an ideal in which both products are identically zero.

(b) $H^{*}(+)$ is closed under the dot product, and it is canonically isomorphic to $\mathcal{A}^{*}$, as an associative algebra.

(c) Let $H(+)^{\sigma}$ be the restricted dual of $H(+)$ defined by the $\bullet$-algebra anti-involution $\sigma$ (section 3.5). Then the ideal $H(-)$ is isomorphic, as module over $H(+)$, to $H(+)^{\sigma}$.

(d) $H^{*}(+)$ is not closed under the bracket product. The sequence in (a) does not split as an exact sequence of graded Lie algebras (For more details on the bracket, see section 3.6).

(e) The projection map $H \longrightarrow \mathcal{A}$ intertwines $-b_{0}$ on $H$ and the differential operator

$$
\Delta=\frac{\partial}{\partial x} \frac{\partial}{\partial x^{*}}+\frac{\partial}{\partial y} \frac{\partial}{\partial y^{*}}
$$

on $\mathcal{A}$ where $x^{*}=\partial_{x}, y^{*}=\partial_{y}$.

\subsection{Discussion}

\subsubsection{Additional examples of string backgrounds}

So far we have discussed in detail the algebraic structure of the $c=1$ model. We will now make some remarks about the 26 dimensional bosonic string background.

In reference [7], the authors consider the string background consisting of 26 bosons compactified on a torus. This may be viewed as a chiral algebra constructed from a 26 dimensional Lorentzian lattice. The chiral BRST cohomology $H$ in this case has the following structure: the ground ring $H^{0}$ consists of only the identity operator. It is also known that the space $P$ of old physical states is isomorphic to $H^{1}$. We show, in section 2.4, that this isomorphism is in fact at the level of Lie algebras, i.e. the Lie bracket on $P$ is compatible with the Gerstenhaber bracket on $H^{1}$. The ghost number two cohomology $H^{2}$ is a module over $H^{1}$. Since $\left\{H^{2}, H^{2}\right\} \subset H^{3}$ and $H^{3}$ is one-dimensional, the bracket provides a bilinear form on $H^{2}$ which is invariant under the $H^{1}$-action. We believe that this new structure is worthy of further study.

In reference [19, we study the backgrounds in which the $c<1$ minimal models are coupled to the Liouville field from the BRST point of view. Since then, many physicists have studied the dot product structure of the BRST cohomology. But we will not attempt to review the recent developments (see for example [14] 15] for references). The full structure of the Gerstenhaber algebras for $c<1$ has not been worked out. Note that we will need some modifications to our theory to take into account the operators with non-integral dimension in the $c<1$ theories. 


\subsubsection{Deformations of a chiral algebra?}

In a recent discussion with Greg Moore, we have learned that deformations of a conformal field theory ought to be connected to BRST invariant operators. The so-called marginal operators correspond to first order deformations (perturbations) of a fixed CFT. The so-called exactly marginal operators give rise to deformations to all orders of the CFT. These deformations occur in the context of a full two-sided CFT. We propose that one should also consider deformations of the corresponding chiral theory.

Given a fixed chiral background $V$ and a dimension zero BRST invariant operator $\phi(z)$, we should consider the associated current $\left(b_{-1} \phi\right)(z)$. The first measure for the failure of $\left(b_{-1} \phi\right)(z)$ to be "marginal" should be indicated by the OPE with itself. Specifically, the first order pole in $\left(b_{-1} \phi\right)(z)\left(b_{-1} \phi\right)(w)$ should represent an obstruction for $\left(b_{-1} \phi\right)(z)$ to be marginal.

Proposition 3.3 The first order pole of the OPE $\left(b_{-1} \phi\right)(z)\left(b_{-1} \phi\right)(w)$ vanishes if and only if the Gerstenhaber bracket $\{\phi, \phi\}$ vanishes.

The above necessary and sufficient condition is very reminiscent of the classical BV equation (on the physical side) [29], and the condition for second order deformation of an associative algebra (on the mathematical side) [12]. It also reminds us of the existence condition for a Poisson structure on a manifold (see Proposition 5.2).

In the $\mathrm{c}=1$ model, let's try to solve the equation

$$
\{\phi, \phi\}=0
$$

We focus on $\phi \in H^{*}(+)$. First observe that by graded skew symmetry of the bracket, the solution set of eqn (3.62) is invariant under the translation by any BRST class $\psi$ with odd ghost number. That is, if $\phi$ is a solution then so is $\phi+\psi$. Since nontrivial states in the $c=1$ model only have ghost number zero through three, it is enough to consider solutions involving ghost number zero and two:

$$
\phi=f+g \cdot \partial_{x} \cdot \partial_{y}
$$

where $f, g$ are polynomial functions to be determined. It is easy to see that eqn (3.62) is now equivalent to the following two equations:

$$
\begin{array}{r}
\left\{f, g \cdot \partial_{x} \cdot \partial_{y}\right\}=0 \\
\left\{g \cdot \partial_{x} \cdot \partial_{y}, g \cdot \partial_{x} \cdot \partial_{y}\right\}=0
\end{array}
$$


Both equations are easy to solved. The most general solution to eqn (3.62) in $H^{*}(+)$ is of one of the following two types (up to translation by odd ghost number state):

(i) $\phi=f$ for any polynomial function $f$;

(ii) $\phi=$ const. $+g \cdot \partial_{x} \cdot \partial_{y}$ for any polynomial function $g$ with zero constant term.

\subsubsection{Modules over the BRST algebra}

As in the case of Hochschild cohomology [12], our theory of Gerstenhaber algebra can be generalized to the case of modules. More precisely, given a chiral background $V$ and a $V$-module $M$ (for definition, see [10]), we can form the corresponding BRST complex:

$$
M \otimes \Lambda^{*}
$$

It can be shown that the correponding cohomology $H^{*}(M)$ is a module over the Gerstenhaber algebra $H^{*}(V)$. This is analogous to the situation in Hochschild theory.

When we pass to BRST cohomology, the space of intertwiners of $V$-modules descends to a space of intertwiners of $H^{*}(V)$-modules.

\subsubsection{Topological chiral algebras}

We can significantly generalize the notion of a string background to the notion of a topological conformal field theory (see for example [5] [6] and references therein).

Definition 3.4 A topological chiral algebra (TCA) consists of the following data: a super chiral algebra $C^{*}$, a weight one even current $F(z)$ whose charge $F_{0}$ is the fermion number operator, a weight one odd primary field $J(z)$ having fermion number one and having a square zero charge $Q$, and a weight two odd primary field $G(z)$ having fermion number -1 and satisfying $[Q, G(z)]=$ $L(z)$ where $L(z)$ is the stress-energy field. We denote the cohomology of the complex $\left(C^{*}, Q\right)$ by $H^{*}(C)$.

Remarkably, all of the structures of our theory generalize to the case of topological chiral algebras. In particular, if we replace the BRST complex by a general TCA $C^{*}$, the ghost number current $c(z) b(z)$ by $F(z)$, the BRST current by $J(z)$, the BRST operator by the charge $Q$ of $J(z)$, and the anti-ghost $b(z)$ by $G(z)$, then the exact translation of Theorem 2.2 holds for $H^{*}(C)$. 
Moreover, the appropriate translations of all the statements in sections 2.1 2.3 hold true in this general context. In particular, we have a coboundary Gerstenhaber algebra on $H^{*}(C)$ (see Definition 5.3), and up to homotopy on $C^{*}$.

Our generalization incorporates many interesting examples. The so-called $\mathrm{N}=2$ twisted super conformal field theories are known to give rise to examples of TCA's (see for example [5] [6]). It can be shown that in these examples, the $G_{0}$ operator acts by zero on cohomology provided the $\mathrm{N}=2$ theory is unitary. As a consequence, by (translation of) Lemma 2.1, the Gerstenhaber bracket is identically zero in cohomology.

Even in the general context of TCA's, the question we raise in section 3.9.2 still makes sense. In particular, what is the relation between the equation $\{\phi, \phi\}=0$ and deformations of $C^{*}$ ? More generally, if $\hbar$ is a parameter, what is the meaning (physical and mathematical) of the "quantum BV" equation:

$$
\hbar G_{0} \phi+\{\phi, \phi\}=0 ?
$$

\subsubsection{Closed string (field) theory}

Up to now, we have restricted our discussion to chiral field theories. In the case of string theory, chiral theories alone are not adequate for describing closed strings [31]35]. At the algebraic level, there are at least two additional things we must do.

First we must tensor the left and the right moving BRST complexes:

$$
C^{*} \otimes \bar{C}^{*}
$$

We observe that this double complex admits all the interesting algebraic structures that the chiral sectors have. However, while the Gerstenhaber bracket on each of the chiral sectors satisfies the Jacobi identity off-shell, the bracket on the double complex does so only up to homotopy. Anyway, the cohomology of the double complex is again a coboundary Gerstenhaber algebra (CGA)(Definition 5.3) which is the tensor product of the left and the right CGA's. The "coboundary operator" is given by $\Delta=b_{0}+\bar{b}_{0}$. Off-shell, the double complex is a CGA up to homotopy.

However, it seems that the proper counterpart of the $b_{0}$ operator is not $b_{0}+\bar{b}_{0}$ in closed string theory [31, but rather it should be $b_{0}^{-}=b_{0}-\bar{b}_{0}$. This means that we should also twist the Gerstenhaber algebra structure on the double complex above by replacing $b_{0}+\bar{b}_{0}$ by $b_{0}-\bar{b}_{0}$. This is equivalent to twisting the Gerstenhaber bracket on $\bar{C}^{*}$ by a minus sign. Thus on the 
double complex, the twisted bracket is given by

$$
(-1)^{|u|}\{u, v\}=b_{0}^{-}(u \cdot v)-\left(b_{0}^{-} u\right) \cdot v-(-1)^{|u|} u \cdot\left(b_{0}^{-} v\right)
$$

where $u, v$ are elements of the double complex. Note that the dot product on the double complex remains compatible with this new bracket.

The notion of a strongly homotopy Lie algebra is well known in mathematics (see LadaStasheff's recent review [16] for an introduction). Recently, this structure has arisen in the context of closed string theory [31]. A similar structure also appears in closed string field theory - both on-shell and off-shell [35]. How are the off-shell SHLA in [31] 35] related to the HLA defined by eqn (3.68)? Are there any higher homotopies (see [16] for definition) in connection with (a)-(e) of our Theorem 2.2? Our work searching for such higher homotopies is underway. We remark that Witten-Zwiebach's construction of the Lie bracket involves only three-point functions, hence may be treated algebraically — just as our construction in eqn (3.68). However, their construction of the higher homotopies requires the consideration of the geometry of moduli spaces. It is therefore interesting to find a precise connection between the the geometric approach and our algebraic approach.

\section{Appendix A}

The main purpose of this section is to show that $H^{*}$ is generated, as a Gerstenhaber algebra, by four generators $x, y, \partial_{x}, \partial_{y}$. Moreover, we describe a basis of $H(-)$, which is dual and complementary to the basis of $H(+)$ given in (3.26), and hence show that $H(-)$ is an ideal with one generator $\left\{\partial_{x}, \partial_{y}\right\}$. The pairing between the two bases is $s l_{2}$ invariant. We show, as a consequence, that $H(-)$ is a subalgebra of $H$ with the zero product. This last assertion can also be drawn from [32], where the product is explicitly computed. We also describe the action of $b_{0}, x, y, \partial_{x}, \partial_{y}$ on $H(-)$ in terms of the above dual basis.

\subsection{The dual basis}

Using eqns (3.22), (3.29), it is easy to get

$$
\begin{aligned}
\left\{\partial_{x}, \partial_{y}\right\} & =c e^{\sqrt{2} \phi} \\
\partial_{x} \cdot \partial_{y} & =-\partial c c e^{\sqrt{2} \phi}
\end{aligned}
$$


With respect to the pairing (3.39), we have

$$
\left\langle\partial_{x} \cdot \partial_{y},\left\{\partial_{x}, \partial_{y}\right\}\right\rangle=-1
$$

Since $\partial_{x}, \partial_{y}$ are self-adjoint, it follows that the four states $\left\{\partial_{x}, \partial_{y}\right\}, \partial_{x} \cdot\left\{\partial_{x}, \partial_{y}\right\}, \partial_{y} \cdot\left\{\partial_{x}, \partial_{y}\right\}$ and $\partial_{x} \cdot \partial_{y} \cdot\left\{\partial_{x}, \partial_{y}\right\}$ are paired with $-\partial_{x} \cdot \partial_{y},-\partial_{y}, \partial_{x}$, and 1 respectively.

To simplify notations, we sometimes write $\left\{\partial_{x}{ }^{n} u\right.$ to mean applying the bracket of $\partial_{x}$ with $u$, n times: $\left\{\partial_{x}, \ldots,\left\{\partial_{x}, u\right\} \ldots\right\}$. Similarly for $\left\{\partial_{y}{ }^{n} u\right.$.

Since $H(-)$ is an ideal containing $\left\{\partial_{x}, \partial_{y}\right\}$, it follows that $x \cdot\left\{\partial_{x}, \partial_{y}\right\}$ must lie in $H(-)$. The state $x \cdot\left\{\partial_{x}, \partial_{y}\right\}$ has quantum numbers $(p, \alpha, g h \#)=(\sqrt{2} / 2, \sqrt{2} / 2,1)$. According to eqn (3.16), this state must be zero. Thus we get, for any polynomial $f$ without a constant term,

$$
f \cdot\left\{\partial_{x}, \partial_{y}\right\}=0
$$

Expanding $0=\left\{\partial_{x}, x \cdot\left\{\partial_{x}, \partial_{y}\right\}\right\}$, we get

$$
x \cdot\left\{\partial_{x},\left\{\partial_{x}, \partial_{y}\right\}\right\}=-\left\{\partial_{x}, x\right\} \cdot\left\{\partial_{x}, \partial_{y}\right\}=-\left\{\partial_{x}, \partial_{y}\right\}
$$

Applying the same trick repeatedly, we get

$$
x \cdot\left\{\partial _ { x } { } ^ { n } \left\{\partial_{y}{ }^{m}\left\{\partial_{x}, \partial_{y}\right\}=-n\left\{\partial _ { x } { } ^ { n - 1 } \left\{\partial_{y}{ }^{m}\left\{\partial_{x}, \partial_{y}\right\}\right.\right.\right.\right.
$$

Similarly, we have

$$
y \cdot\left\{\partial _ { x } { } ^ { n } \left\{\partial_{y}{ }^{m}\left\{\partial_{x}, \partial_{y}\right\}=-m\left\{\partial _ { x } { } ^ { n } \left\{\partial_{y}{ }^{m-1}\left\{\partial_{x}, \partial_{y}\right\}\right.\right.\right.\right.
$$

This shows that the $\left\{\partial_{x}{ }^{n}\left\{\partial_{y}{ }^{m}\left\{\partial_{x}, \partial_{y}\right\}\right.\right.$ are all nonzero because we can hit them with $x, y$ repeatedly to get down to a nonzero multiple of $\left\{\partial_{x}, \partial_{y}\right\}$. Moreover, they all have distinct quantum numbers, and hence are linearly independent. In fact, by comparing them with the quantum number spectrum given by Theorem 3.1, we see that they form a basis of $H^{1}(-)$.

We can repeat the above argument, with $\left\{\partial_{x}, \partial_{y}\right\}$ replaced by $\partial_{x} \cdot\left\{\partial_{x}, \partial_{y}\right\}, \partial_{y} \cdot\left\{\partial_{x}, \partial_{y}\right\}$ or $\partial_{x} \cdot \partial_{y} \cdot\left\{\partial_{x}, \partial_{y}\right\}$. Then eqns (4.5), (4.6), with the appropriate changes, still hold. Again we get some bases for $H^{2}(-)$ and $H^{3}(-)$. In fact, by comparing quantum numbers, one can easily see that the new bases we obtained can be identified with the bases introduced in [31]. The identification goes as follows: for nonnegative half integer $s$, and $n=-s,-s+1, \ldots, s$,

$$
\begin{aligned}
Y_{s, n}^{-} \sim & \left\{\partial _ { x } { } ^ { s - n } \left\{\partial_{y}{ }^{s+n}\left(\left\{\partial_{x}, \partial_{y}\right\}\right)\right.\right. \\
a Y_{s, n}^{-} \sim & (s-n)\left\{\partial _ { x } { } ^ { s - n - 1 } \left\{\partial_{y}{ }^{s+n}\left(\partial_{x} \cdot\left\{\partial_{x}, \partial_{y}\right\}\right)\right.\right. \\
& +(s+n)\left\{\partial _ { x } { } ^ { s - n } \left\{\partial_{y}{ }^{s+n-1}\left(\partial_{y} \cdot\left\{\partial_{x}, \partial_{y}\right\}\right)\right.\right. \\
P_{s, n} \sim & \left\{\partial _ { x } { } ^ { s - n + 1 } \left\{\partial_{y}{ }^{s+n}\left(\partial_{y} \cdot\left\{\partial_{x}, \partial_{y}\right\}\right)\right.\right. \\
& -\left\{\partial _ { x } { } ^ { s - n } \left\{\partial_{y}{ }^{s+n+1}\left(\partial_{x} \cdot\left\{\partial_{x}, \partial_{y}\right\}\right)\right.\right. \\
a P_{s, n} \sim & \left\{\partial _ { x } { } ^ { s - n } \left\{\partial_{y}{ }^{s+n}\left(\partial_{x} \cdot \partial_{y} \cdot\left\{\partial_{x}, \partial_{y}\right\}\right)\right.\right.
\end{aligned}
$$


This shows that $H(-)$, as an ideal of the Gerstenhaber algebra $H$, is generated by $\left\{\partial_{x}, \partial_{y}\right\}$. For completeness, let's write down a basis for $H(+)$ as well:

$$
\begin{aligned}
a Y_{s, n}^{+} & \sim x^{s-n} \cdot y^{s+n} \cdot \partial_{x} \cdot \partial_{y} \\
Y_{s, n}^{+} & \sim \partial_{x}\left(x^{s-n} \cdot y^{s+n}\right) \cdot \partial_{y}-\partial_{y}\left(x^{s-n} \cdot y^{s+n}\right) \cdot \partial_{x} \\
a \mathcal{O}_{s, n} & \sim x^{s-n} \cdot y^{s+n} \cdot\left(x \cdot \partial_{x}+y \cdot \partial_{y}\right) \\
\mathcal{O}_{s, n} & \sim x^{s-n} \cdot y^{s+n}
\end{aligned}
$$

\subsection{The $s l_{2}$ action}

What can we say about these bases? Already it has been indicated in [28] 31] that for fixed $s$, each of the multiplets $(n=-s,-s+1, \ldots, s)$ in eqns (4.11)- (4.14) is an $s l_{2}$ spin $s$ multiplet. One can represent the $s l_{2}$ generators simply by $\left\{x \cdot \partial_{y}, *\right\},\left\{x \cdot \partial_{x}-y \cdot \partial_{y}, *\right\}$ and $\left\{y \cdot \partial_{x}, *\right\}$. Computing the action of these $s l_{2}$ generators on (4.11)- (4.14) is straightforward, because the relations (3.55) (4.3) suffice. But computing the action on those multiplets in eqns (4.7)- (4.10) is more difficult because the relations (3.55) (4.3) alone are not enough.

Fortunately, we can once again use the pairing between $H(+)$ and $H(-)$. It is easy to check that for any $u, v$ in $H$, and any one of the $s l_{2}$ generators $\{X, *\}$ above, we have

$$
\langle\{X, u\}, v\rangle=\langle u,-\{X, v\}\rangle
$$

i.e. the pairing is $s l_{2}$ invariant. To see that each of the multiplets in the $H(-)$ sector - eqns (4.7)- (4.10) (fixed $s$ and $n=-s,-s+1, \ldots, s$ ) - is indeed an $s l_{2}$ spin $s$ multiplet, it is enough to show that it pairs with a multiplet of the same spin in the $H(+)$ sector. Now using the adjointness property (3.34) of $x, y, \partial_{x}, \partial_{y}$, and using the dot products of $x, y$ with each of the states in eqns (4.7)- (4.10), we obtain the orthogonality relations:

$$
\begin{aligned}
& \left\langle x^{s-n} \cdot y^{s+n} \cdot \partial_{x} \cdot \partial_{y},\left\{\partial_{x}{ }^{s-n}\left\{\partial_{y}{ }^{s+n}\left(\left\{\partial_{x}, \partial_{y}\right\}\right)\right\rangle\right.\right. \\
& =-(s-n) !(s+n) ! \\
& \left\langle\partial_{x}\left(x^{s-n} \cdot y^{s+n}\right) \cdot \partial_{y}-\partial_{y}\left(x^{s-n} \cdot y^{s+n}\right) \cdot \partial_{x},\right. \\
& (s-n)\left\{\partial _ { x } { } ^ { s - n - 1 } \left\{\partial_{y}{ }^{s+n}\left(\partial_{x} \cdot\left\{\partial_{x}, \partial_{y}\right\}\right)+(s+n)\left\{\partial_{x}{ }^{s-n}\left\{\partial_{y}{ }^{s+n-1}\left(\partial_{y} \cdot\left\{\partial_{x}, \partial_{y}\right\}\right)\right\rangle\right.\right.\right. \\
& =-2 s(s-n) !(s+n) ! \\
& \left\langle x^{s-n} \cdot y^{s+n} \cdot\left(x \cdot \partial_{x}+y \cdot \partial_{y}\right),\right. \\
& \left\{\partial _ { x } { } ^ { s - n + 1 } \left\{\partial_{y}{ }^{s+n}\left(\partial_{y} \cdot\left\{\partial_{x}, \partial_{y}\right\}\right)-\left\{\partial_{x}{ }^{s-n}\left\{\partial_{y}{ }^{s+n+1}\left(\partial_{x} \cdot\left\{\partial_{x}, \partial_{y}\right\}\right)\right\rangle\right.\right.\right. \\
& =(2 s+2)(s-n) !(s+n) !
\end{aligned}
$$




$$
\begin{aligned}
& \left\langle x^{s-n} \cdot y^{s+n},\left\{\partial_{x}{ }^{s-n}\left\{\partial_{y}^{s+n}\left(\partial_{x} \cdot \partial_{y} \cdot\left\{\partial_{x}, \partial_{y}\right\}\right)\right\rangle\right.\right. \\
& =(s-n) !(s+n) !
\end{aligned}
$$

All the other inner products are zero. This proves our assertion.

\subsection{The dot product in $H(-)$}

Since there is no state with ghost number greater than 3 , the only possibly nonzero dot products that we need to consider are those on $H^{1}(-) \times H^{1}(-)$ and $H^{1}(-) \times H^{2}(-)$.

Consider the product in $H^{1}(-)$, which is spanned by the $Y_{s, n}^{-}$. Obviously, the $s l_{2}$ action considered above acts by derivations of the dot product. This means that the product of two states $Y_{s, n}^{-}, Y_{s^{\prime}, n^{\prime}}^{-}$(see eqn (4.7)), must lie in the tensor product representation of those two spins. In particular, it must be a linear combination of states with spin $s^{\prime \prime}$ satisfying $\left|s-s^{\prime}\right| \leq s^{\prime \prime} \leq s+s^{\prime}$. Now with this restriction and by the conservation of quantum numbers (i.e. $p, \alpha, g h \#$ ), we must have

$$
Y_{s, n}^{-} \cdot Y_{s^{\prime}, n^{\prime}}^{-}=\text {constant } P_{s+s^{\prime}, n+n^{\prime}}
$$

To prove that this is zero, it is enough to show that the inner product of the left hand side with $a \mathcal{O}_{s+s^{\prime}, n+n^{\prime}}$ (see eqn (4.13)) is zero. Consider

$$
\begin{aligned}
& \left\langle a \mathcal{O}_{s+s^{\prime}, n+n^{\prime}}, Y_{s, n}^{-} \cdot Y_{s^{\prime}, n^{\prime}}^{-}\right\rangle \\
& \sim\left\langle x^{s+s^{\prime}-n-n^{\prime}+1} \cdot y^{s+s^{\prime}+n+n^{\prime}} \cdot \partial_{x}, Y_{s, n}^{-} \cdot Y_{s^{\prime}, n^{\prime}}^{-}\right\rangle \\
& +\left\langle x^{s+s^{\prime}-n-n^{\prime}} \cdot y^{s+s^{\prime}+n+n^{\prime}+1} \cdot \partial_{y}, Y_{s, n}^{-} \cdot Y_{s^{\prime}, n^{\prime}}^{-}\right\rangle
\end{aligned}
$$

Since $x, y$ are anti-selfadjoint, we can bring their monomials to the second slot, with only some sign change. Because of eqn (4.5) and the fact that $s+s^{\prime}-n-n^{\prime}+1>s-n$, we have $x^{s+s^{\prime}-n-n^{\prime}+1} \cdot Y_{s, n}^{-}=0$. Similarly, $y^{s+s^{\prime}+n+n^{\prime}+1} \cdot Y_{s^{\prime}, n^{\prime}}^{-}=0$. This shows that $H^{1}(-) \cdot H^{1}(-)=0$. The argument for $H^{1}(-) \cdot H^{2}(-)=0$ is the same.

\section{$4.4 b_{0}, x, y, \partial_{x}, \partial_{y}$ acting on $H(-)$}

Finally, we would like to give a more explicit description of $H(-)$ as a module over the Gerstenhaber algebra $H$. We should therefore describe - in terms of a nice basis - how the generators $x, y, \partial_{x}, \partial_{y}$ of $H$ act on $H(-)$, first by the dot product, then by the bracket. 
The actions of $x, y$ on the basis (4.7)- 4.10) of $H(-)$ have already been alluded to above. We summarize them as follows. Let $\lambda$ be one of the following four special states $\left\{\partial_{x}, \partial_{y}\right\}, \partial_{x}$. $\left\{\partial_{x}, \partial_{y}\right\}, \partial_{y} \cdot\left\{\partial_{x}, \partial_{y}\right\}$ or $\partial_{x} \cdot \partial_{y} \cdot\left\{\partial_{x}, \partial_{y}\right\}$. Then we have

$$
\begin{aligned}
& x \cdot\left\{\partial _ { x } { } ^ { n } \left\{\partial_{y}{ }^{m} \lambda=-n\left\{\partial _ { x } { } ^ { n - 1 } \left\{\partial_{y}{ }^{m} \lambda\right.\right.\right.\right. \\
& y \cdot\left\{\partial _ { x } { } ^ { n } \left\{\partial_{y}{ }^{m} \lambda=-m\left\{\partial _ { x } { } ^ { n } \left\{\partial_{y}{ }^{m-1} \lambda\right.\right.\right.\right.
\end{aligned}
$$

To work out the action of $\partial_{x}$, we note for example that

$$
\begin{aligned}
\partial_{x} \cdot\left\{\partial_{y}, \lambda\right\} & =\left\{\partial_{y}, \partial_{x} \cdot \lambda\right\}-\left\{\partial_{y}, \partial_{x}\right\} \cdot \lambda \\
& =\left\{\partial_{y}, \partial_{x} \cdot \lambda\right\}
\end{aligned}
$$

This holds because $\left\{\partial_{y}, \partial_{x}\right\}$ and $\lambda$ are both in $H(-)$, and hence have zero dot product. Similarly, we have

$$
\begin{aligned}
& \partial_{x} \cdot\left\{\partial _ { x } { } ^ { n } \left\{\partial_{y}{ }^{m} \lambda=\left\{\partial _ { x } { } ^ { n } \left\{\partial_{y}{ }^{m}\left(\partial_{x} \cdot \lambda\right)\right.\right.\right.\right. \\
& \partial_{y} \cdot\left\{\partial _ { x } { } ^ { n } \left\{\partial_{y}{ }^{m} \lambda=\left\{\partial _ { x } { } ^ { n } \left\{\partial_{y}{ }^{m}\left(\partial_{y} \cdot \lambda\right)\right.\right.\right.\right.
\end{aligned}
$$

In section 2.3, we proved that $b_{0}$ acts as a derivation of the bracket in $H$. Using this and the fact that $b_{0}$ kills both $\partial_{x}, \partial_{y}$, we get

$$
\begin{aligned}
b_{0}\left\{\partial _ { x } { } ^ { n } \left\{\partial_{y}{ }^{m}\left(\left\{\partial_{x}, \partial_{y}\right\}\right)=\right.\right. & 0 \\
b_{0}\left\{\partial _ { x } { } ^ { n } \left\{\partial_{y}{ }^{m}\left(\partial_{x} \cdot\left\{\partial_{x}, \partial_{y}\right\}\right)=\right.\right. & -\left\{\partial _ { x } { } ^ { n + 1 } \left\{\partial_{y}{ }^{m}\left(\left\{\partial_{x}, \partial_{y}\right\}\right)\right.\right. \\
b_{0}\left\{\partial _ { x } { } ^ { n } \left\{\partial_{y}{ }^{m}\left(\partial_{y} \cdot\left\{\partial_{x}, \partial_{y}\right\}\right)=\right.\right. & -\left\{\partial _ { x } { } ^ { n } \left\{\partial_{y}{ }^{m+1}\left(\left\{\partial_{x}, \partial_{y}\right\}\right)\right.\right. \\
b_{0}\left\{\partial _ { x } { } ^ { n } \left\{\partial_{y}{ }^{m}\left(\partial_{x} \cdot \partial_{y} \cdot\left\{\partial_{x}, \partial_{y}\right\}\right)=\right.\right. & -\left\{\partial _ { x } { } ^ { n + 1 } \left\{\partial_{y}{ }^{m}\left(\partial_{y} \cdot\left\{\partial_{x}, \partial_{y}\right\}\right)\right.\right. \\
& +\left\{\partial _ { x } { } ^ { n } \left\{\partial_{y}{ }^{m+1}\left(\partial_{x} \cdot\left\{\partial_{x}, \partial_{y}\right\}\right)\right.\right.
\end{aligned}
$$

We can now summarize the bracket operations of $x, y, \partial_{x}, \partial_{y}$ with $H(-)$ :

$$
\begin{aligned}
\left\{\partial_{x},\left(\left\{\partial_{x}{ }^{n}\left\{\partial_{y}{ }^{m} \lambda\right)\right\}\right.\right. & =\left\{\partial _ { x } { } ^ { n + 1 } \left\{\partial_{y}{ }^{m} \lambda\right.\right. \\
\left\{\partial_{y},\left(\left\{\partial_{x}{ }^{n}\left\{\partial_{y}{ }^{m} \lambda\right)\right\}\right.\right. & =-\left\{\partial _ { x } { } ^ { n } \left\{\partial_{y}{ }^{m+1} \lambda\right.\right. \\
\left\{x,\left(\left\{\partial_{x}{ }^{n}\left\{\partial_{y}{ }^{m}\left(\left\{\partial_{x}, \partial_{y}\right\}\right)\right)\right\}\right.\right. & =0 \\
\left\{y,\left(\left\{\partial_{x}{ }^{n}\left\{\partial_{y}{ }^{m}\left(\left\{\partial_{x}, \partial_{y}\right\}\right)\right)\right\}\right.\right. & =0 \\
\left\{x,\left(\left\{\partial_{x}{ }^{n}\left\{\partial_{y}{ }^{m}\left(\partial_{x} \cdot\left\{\partial_{x}, \partial_{y}\right\}\right)\right)\right\}\right.\right. & =-\left\{\partial _ { x } { } ^ { n } \left\{\partial_{y}{ }^{m}\left(\left\{\partial_{x}, \partial_{y}\right\}\right)\right.\right. \\
\left\{y,\left(\left\{\partial_{x}{ }^{n}\left\{\partial_{y}{ }^{m}\left(\partial_{x} \cdot\left\{\partial_{x}, \partial_{y}\right\}\right)\right)\right\}\right.\right. & =0 \\
\left\{x,\left(\left\{\partial_{x}{ }^{n}\left\{\partial_{y}{ }^{m}\left(\partial_{y} \cdot\left\{\partial_{x}, \partial_{y}\right\}\right)\right)\right\}\right.\right. & =0
\end{aligned}
$$




$$
\begin{aligned}
\left\{y,\left(\left\{\partial_{x}{ }^{n}\left\{\partial_{y}{ }^{m}\left(\partial_{y} \cdot\left\{\partial_{x}, \partial_{y}\right\}\right)\right)\right\}\right.\right. & =-\left\{\partial _ { x } { } ^ { n } \left\{\partial_{y}{ }^{m}\left(\left\{\partial_{x}, \partial_{y}\right\}\right)\right.\right. \\
\left\{x,\left(\left\{\partial_{x}{ }^{n}\left\{\partial_{y}{ }^{m}\left(\partial_{x} \cdot \partial_{y} \cdot\left\{\partial_{x}, \partial_{y}\right\}\right)\right)\right\}\right.\right. & =-\left\{\partial _ { x } { } ^ { n } \left\{\partial_{y}{ }^{m}\left(\partial_{y} \cdot\left\{\partial_{x}, \partial_{y}\right\}\right)\right.\right. \\
\left\{y,\left(\left\{\partial_{x}{ }^{n}\left\{\partial_{y}{ }^{m}\left(\partial_{x} \cdot \partial_{y} \cdot\left\{\partial_{x}, \partial_{y}\right\}\right)\right)\right\}\right.\right. & =\left\{\partial _ { x } { } ^ { n } \left\{\partial_{y}{ }^{m}\left(\partial_{x} \cdot\left\{\partial_{x}, \partial_{y}\right\}\right)\right.\right.
\end{aligned}
$$

where $\lambda$ is any one of the four special states.

\section{Appendix B}

In this section, we will make a few introductory comments about the mathematical theory of Gerstenhaber algebras; for a more mathematically advanced and complete account of the theory see the forthcoming preprint [34].

Definition 5.1 A Gerstenhaber algebra $G^{*}$ is a $\mathbf{Z}$-graded vector space equipped with two bilinear multiplication operations, denoted by $u \cdot v$ and $\{u, v\}$ respectively, and satisfying the following assumptions:

(i) If $u$ and $v$ are homogeneous elements of degree $|u|$ and $|v|$ respectively, then $u \cdot v$ is homogeneous of degree $|u|+|v|$ and $\{u, v\}$ is homogeneous of degree $|u|+|v|-1$.

(ii) Identities (a) through (e) from Theorem 2.9 above hold for any triple of homogeneous elements $u, v$ and $t$ in $G^{*}$.

Note: We will call the product $u \cdot v$ the dot product and the product $\{u, v\}$ the Gerstenhaber bracket product.

\section{Remarks:}

(i) Physicists will want to call the degree of a homogeneous element the ghost number. Mathematicians are primarily familiar with examples in which the degree is bounded from above or from below by zero.

(ii) With respect to the dot product, $G^{*}$ is a $\mathbf{Z}$-graded supercommutative associative algebra.

(iii) Let $\Pi G^{*}$ be the graded vector space defined by $\Pi G^{k}=G^{k-1}$. Then, with respect to the bracket product, $\Pi G^{*}$ is a $\mathbf{Z}$-graded Lie superalgebra.

(iv) Identity (e) in Theorem 2.2 establishes the crucial link between the two different products in $G^{*}$.

The following are a few classes of examples of a Gerstenhaber algebra. 
A). The simplest Gerstenhaber algebras are the following: let $A_{n}^{*}$ be the $\mathbf{Z}$-graded supercommutative algebra generated by $n$ variables $x_{1}, x_{2}, \ldots, x_{n}$, of degree zero, and $n$ variables $\partial_{x_{1}}, \partial_{x_{2}}, \ldots, \partial_{x_{n}}$, of degree one. We refer to an element of this algebra as a polyvector field. The elements of degree zero are functions, the elements of degree one are vector fields, of degree two are bivector fields, and so on. The dot product is simply the graded commutative multiplication of polyvector fields.

Long ago, Schouten [24] defined a bracket operation on polyvector fields (he thought of such fields as antisymmetric contravariant tensor fields.) The Schouten bracket is characterized by the following:

(i) For any two function $f$ and $g,\{f, g\}=0$.

(ii) If $f$ is a function and $X$ is a vector field, $\{X, f\}=-\{f, X\}=X f$, i.e. the evaluation of the vector field $X$ on the function $f$.

(iii) If $X$ and $Y$ are vector fields, then $\{X, Y\}=[X, Y]$, the Lie bracket of the vector fields.

(iv) Together, the dot product and the Schouten bracket endow $A_{n}^{*}$ with structure of a Gerstenhaber algebra. In particular, identity (e) holds.

\section{Remarks:}

(i) It is an elementary and useful exercise to write an explicit formula for the Schouten bracket of any two polyvector fields.

(ii) The algebra $A_{n}^{*}$ appears in Witten's article [29], where the variables $\partial_{x_{k}}$ are denoted by $x_{k}^{*}$, $k=1, \ldots, n$, and the Schouten bracket is defined as a kind of Poisson bracket with unusual signs - more precisely, an odd-Poisson bracket. (The sign convention we use here differs slightly from the one in [29.) Thus the algebra $A_{n}^{*}$ arises naturally in the theory of Batalin and Vilkovisky, who employ a field space generalization of the algebras $A_{n}^{*}$. We will say more about $A_{n}^{*}$ and $\mathrm{BV}$ theory below.

B). For a different class of Gerstenhaber algebras, let $g$ be any Lie algebra, and let $\Lambda^{*} g$ be the Grassmann algebra generated by $g$. We can define a bracket $\{$,$\} on \Lambda^{*} g$ by requiring the following:

(i) If $a$ and $b$ are scalars, $\{a, b\}=0$.

(ii) If $X$ is in $g$ and $a$ is a scalar, then $\{X, a\}=0$.

(iii) If $X$ and $Y$ are in $g$, then $\{X, Y\}=[X, Y]$, the Lie bracket in $g$.

(iv) The wedge product together with the bracket product endow $\Lambda^{*} g$ with the structure of a Gerstenhaber algebra. In particular, identity (e) above holds. 


\section{Remarks:}

(i) The algebra $\Lambda^{*} g$ is discussed briefly in Drinfeld's famous article on quantum groups [4]. However, Drinfeld mistakenly implies that if $V_{n}$ is the Lie algebra of polynomial coefficient vector fields in $n$ variables, then $\Lambda^{*} V_{n}$ is isomorphic as a Gerstenhaber algebra to $A_{n}^{*}$. In fact, there is a Gerstenhaber algebra homomorphism from $\Lambda^{*} V_{n}$ to $A_{n}^{*}$ which is an isomorhism at the degree one level but which clearly fails to be an isomorphism at degree zero, since $\Lambda^{0} V_{n}$ is just one dimensional.

(ii) It is an elementary exercise to write the explicit bracket of two exterior forms in the algebra $\Lambda^{*} g$, for arbitrary $g$.

(iii) If $g$ is finite dimensional, $\Lambda^{*} g$ and $\Lambda^{*} h$ are isomorphic as Gerstenhaber algebras if and only if $g$ and $h$ are isomorphic as Lie algebras.

C). A more sophisticated class of Gerstenhaber algebras arise as follows: Let $M$ ba a manifold (differentiable, complex, algebraic, etc.). Let $F(M)$ be the commutative algebra of functions (of the appropriate type - differentiable, holomorphic, algebraic, etc.) on $M$. Let $G^{*}(M)$ be the algebra of polyvector fields on $M$, with the operations of wedge product and the Schouten bracket, defined by anology with the bracket in $A_{n}^{*}$. Then $G^{*}(M)$ is a Gerstenhaber algebra.

The algebras $G^{*}(M)$ can be thought of in the context of BV theory: we can regard $G^{*}(M)$ as the commutative superalgebra of functions on $\Pi T^{*} M$, the cotangent bundle of $M$ with the fibers made into odd supervector spaces. The Gerstenhaber bracket in $G^{*}(M)$ is the odd Poisson bracket associated to the canonical odd symplectic two-form on $\Pi T^{*} M$.

We should mention an important application of the algebras, $G^{*}(M)$ : Let $P$ be a bivector field on $M$. We can always construct a bracket operation on the algebra $F(M)$ by the formula

$$
\{f, g\}_{P}=\iota(P)(d f \wedge d g)
$$

where $\iota(P)$ denotes contraction of $P$ against a two-form. The question is, when does this new bracket $\{,\}_{P}$ give rise to a Lie algebra structure on $F(M)$ ?

Proposition $5.2\{,\}_{P}$ satisfies the Jacobi identity if and only if the Schouten bracket $\{P, P\}=$ 0 .

Note that when our bracket on functions satisfies Jacobi, the algebra $F(M)$ becomes what is known in mathematical physics as a Poisson algebra (see for example [4]).

D). Let $N$ be a super-manifold with an odd symplectic structure. Then the supercommutative algebra of functions $F(N)$ on $N$ has a structure of a $\mathbf{Z} / 2$-graded, rather than $\mathbf{Z}$-graded 
Gerstenhaber algebra. We now have the most general context of BV theory, as described in [30]. The classical BV master equation reads: $\{S, S\}=0$ for some even function $S$ in $F(N)$. We see from Proposition 5.2 that the theory of Poisson bracket structures on function algebras bears a close relation to $\mathrm{BV}$ theory.

E). The abstract notion of a Gerstenhaber algebra first arose in work by M.Gerstenhaber on the Hochschild cohomology of an associative algebra [11] [12] [13] [25]. In the very special case that the associative algebra is of the form $F(M)$ for some smooth manifold $M$, it is a theorem that the Hochschild cohomology of $F(M)$ is isomorphic as a Gerstenhaber algebra to the algebra $G^{*}(M)$ defined in example $\mathrm{C}$ ) above. However, in general the Hochschild cohomology of an associative algebra is known to be non-isomorphic to an algebra of type $G^{*}(M)$ (see [13]).

Important Remark: Just as Poisson algebras are far reaching generalizations of Poisson algebra of functions on the phase space of classical mechanics - i.e. symplectic vector space - Gerstenhaber algebras are far reaching generalizations of the elementary examples in A) through $\mathrm{C}$ ) above. There is an overlap with BV theory, as we explained in the above, but it would be quite misleading to identify Gerstenhaber algebra theory with BV theory.

F). Coboundary Gerstenhaber algebras: in the context of our Theorem 2.2, we have proved the following (see Lemma 2.1):

On the BRST complex, the following identity holds:

$$
(-1)^{|u|}\{u, v\}=b_{0}(u \cdot v)-\left(b_{0} u\right) \cdot v-(-1)^{|u|} u \cdot\left(b_{0} v\right)
$$

Thus, the bracket on the BRST complex measures the failure of the operator $b_{0}$ to be a derivation of the dot product. The above statement is the precise analog of an observation made by Witten in the context of BV theory. Consider again the algebra $A_{n}^{*}$, and define a differential operator $\Delta$ in $A_{n}^{*}$ by the formula:

$$
\Delta=\sum_{i} \frac{\partial}{\partial x_{i}} \frac{\partial}{\partial x_{i}^{*}}
$$

Then the Schouten bracket in $A_{n}^{*}$ measures the failure of $\Delta$ to be a derivation of the dot product in $A_{n}^{*}$. We propose the following abstract definition:

Definition 5.3 Let $G^{*}$ be a $\mathbf{Z}$-graded Gerstenhaber algebra with a linear operator $\Delta$ of degree -1 such that the following identity holds:

$$
(-1)^{|u|}\{u, v\}=\Delta(u \cdot v)-(\Delta u) \cdot v-(-1)^{|u|} u \cdot(\Delta v)
$$

Then we call the pair $\left(G^{*}, \Delta\right)$ a coboundary Gerstenhaber algebra. 


\section{Remarks:}

(i) The motivation for our terminology is simple: the above equation expresses the fact that (up to certain signs) the bracket, as a bilinear operator, is the Hochschild coboundary [12] of the linear operator $\Delta$.

(ii) A given Gerstenhaber algebra $G^{*}$ can be a coboundary algebra in more than one way: given a $\Delta$ operator as above, we can simply add any derivation of degree -1 to $\Delta$. Witten analyzes the case of $G^{*}(M)$ when $M$ is a manifold, and observes that any volume form $\Omega$ on $M$ gives rise to a natural $\Delta(\omega)$ operator such that $\left(G^{*}(M), \Delta(\omega)\right)$ is a coboundary algebra. It is easy to check

that for two different volume forms, the corresponding delta operators differ by a derivation of degree -1 .

(iii) For any Lie algebra $g$, the Gerstenhaber algebra $\Lambda^{*} g$ is canonically a coboundary algebra: for the delta operator we may take the Lie algebra homology differential, $\partial$ (see [34]).

(iv) If $M$ is a manifold or variety with singularities, we can still define the Gerstenhaber algebra $G^{*}(M)$, but it is not clear under which circumstances $G^{*}(M)$ is of coboundary type, since we cannot simply appeal to the existence of a volume form.

(v) If $N$ is an odd symplectic supermanifold, it is again not clear whether we have a global delta operator making $F(N)$ into a coboundary $\mathbf{Z} / 2$-graded Gerstenhaber algebra. BV theory appears to require the existence of such a delta operator.

(vi) If $A$ is an associative algebra, it is not known under what conditions that Hochschild cohomology of $A$ is a coboundary Gerstenhaber algebra. The theorem alluded to in E) tells us that if $A$ is $F(M)$ for a smooth manifold or variety, then the Hochschild cohomology is in this case a coboundary algebra.

\section{References}

[1] P.Bouwknegt, J.McCarthy and K.Pilch, Commun. Math. Phys. 145 (1992) 541.

[2] A. Belavin, A.M. Polyakov and A.A. Zamolodchikov, "Infinite conformal symmetry in two dimensional quantum field theory", Nucl. Phys. B241 (1984) 333.

[3] R.E. Borcherds, "Vertex operator algebras, Kac-Moody algebras and the Monster", Proc. Natl. Acad. Sci. USA. 83 (1986) 3068.

[4] V.G. Drinfel'd, "Quantum Groups", ICM Proceedings, Berkeley, California, USA (1986) 798. 
[5] R. Dijkgraaf, H. Verlinde and E. Verlinde, "Notes on topological string theory and 2d quantum gravity", especially references therein, preprint PUPT-1217, IASSNS-HEP-90/80.

[6] T. Eguchi and S.K. Yang, " $\mathrm{N}=2$ superconformal models as topological field theories", Tokyo-preprint UT-564.

[7] I.B. Frenkel, H. Garland and G.J. Zuckerman, "Semi-infinite cohomology and string theory", Proc. Natl. Acad. Sci. USA 83 (1986) 8442.

[8] E.Frenkel, "Determinant formulas for the free field representations of the Virasoro and Kac-Moody algebras", Phys. Lett. 285B (1992) 71.

[9] I.B. Frenkel, J. Lepowsky and A. Meurman, "Vertex Operator Algebras and the Monster", Academic Press, New York, 1988.

[10] I.B. Frenkel, Y. Huang and J. Lepowsky, "On axiomatic approaches to vertex operator algebras and modules", Yale-Rutgers preprint, Dept. of Math., 1989.

[11] M. Gerstenhaber, "The cohomology structure of an associative ring", Ann. of Math. 78, No.2 (1962) 267.

[12] M. Gerstenhaber, "On the deformation of rings and algebras", Ann. of Math. 79, No.1 (1964) 59.

[13] M. Gerstenhaber, A. Giaquinto and S. Schack, "Quantum symmetry", Preprint.

[14] H. Kanno and M.H. Sarmadi, "BRST cohomology ring in 2d gravity coupled to minimal models", preprint hepth9207078.

[15] D. Kutasov, E. Martinec and N. Seiberg, Phys. Lett. B276 (1992) 437.

[16] T. Lada and J. Stasheff, "Introduction to sh Lie algebras for physicists", preprint UNCMATH-92/2.

[17] B.H. Lian, "Semi-infinite homology and 2d quantum gravity", Dissertation, Yale Univ. May 1991.

[18] B.H. Lian, "On the classification of simple vertex operator algebras", Univ. of Toronto preprint, February 1992.

[19] B.H. Lian and G.J. Zuckerman, "New selection rules and physical states in 2d gravity; conformal gauge", Phys. Lett B, 254 , No.3,4 (1991) 417. 
[20] B.H. Lian and G.J. Zuckerman, "2d gravity with c=1 matter",Phys. Lett. B266 (1991) 21.

[21] B.H. Lian and G.J. Zuckerman, "Semi-infinite homology and 2D gravity (I)", Commun. Math. Phys. 145 (1992) 561.

[22] G. Moore and N. Seiberg, "Classical and quantum conformal field theory", Commun. Math. Phys. 123 (1989) 177-254.

[23] S.Mukherji, S.Mukhi and A.Sen, Phys. Lett 266B (1991)337.

[24] J.A. Schouten, "Ueber Differentialkomitanten zweier kontravarianter Grossen", Nederl. Acad. Wetensch. Proc. Ser. A 43 (1940) 449-452.

[25] M. Schlesinger and J.Stasheff, "The Lie algebra structure of tangent cohomology and deformation theory", J. Pure Appl. Alg. 38 (1985) 313.

[26] J.Stasheff, "Differential graded Lie algebras, quasi-Hopf algebras and higher homotopy algebras", UNC Math preprint, 1991.

[27] J.Stasheff, "Homotopy associativity of H-spaces. I", AMS Trans. 108 (1963) 275.

[28] E. Witten, "Ground ring of the two dimensional string theory", Nucl. Phys. B373 (1992) 187.

[29] E. Witten, "The anti-bracket formalism", preprint IASSNS-HEP-90/9.

[30] E. Witten, "On background independent open-string field theory", preprint FERMILABPUB-IASSNS-HEP-92/53.

[31] E. Witten and B. Zwiebach, "Algebraic structures and differential geometry in 2d string theory", Nucl. Phys. B377 (1992) 55.

[32] Y. Wu and C. Zhu, "The complete structure of the cohomology ring and associated symmetries in $\mathrm{d}=2$ string theory", preprint.

[33] Y. Zhu, "Vertex operator algebras, elliptic functions and modular forms", Yale Thesis, Dept. of Math., 1990.

[34] G.J. Zuckerman, "New applications of Gerstenhaber algebras", in progress.

[35] B. Zwiebach, "Closed string field theory: quantum action and the B-V master equation", preprint IASSNS-HEP-92/41. 